\title{
Planetary Cratering Mechanics
}

\author{
JoHn D. O' KeEFE AND Thomas J. AHRENS
}

Lindhurst Laboratory of Experimental Geophysics, Seismological Labonatory, Califomia Institute of Technology, Pasadena

\begin{abstract}
The objective of this study was to obtain a quantitative understanding of the cratering process over a broad range of conditions. Our approach was to numerically compute the evolution of impact induced flow fields and calculate the time histories of the key measures of crater geometry (e.g. depth, diameter, lip height) for variations in planetary gravity $\left(0\right.$ to $10^{9} \mathrm{~cm} / \mathrm{s}^{2}$ ), material strength (0 to $2400 \mathrm{kbar}$ ), and impactor radius (0.05 to $5000 \mathrm{~km}$ ). These results were used to establish the values of the open parameters in the scaling laws of Holsapple and Schmidt (1987). We describe the impact process in terms of four regimes: (1) penetration, (2) inertial, (3) terminal and (4) relaxation. During the penetration regime, the depth of impactor penetration grows linearly for dimensionless times $\tau=(U t / a)<5.1$. Here, $U$ is projectile velocity, $t$ is time, and $a$ is projectile radius. In the inertial regime, $\tau>5.1$, the crater grows at a slower rate until it is arrested by either strength or gravitational forces. In this regime, the increase of crater depth, $d$, and diameter, $D$, normalized by projectile radius is given by $d / a=1.3(U t / a)^{0.36}$ and $D / a=2.0(U t / a)^{0.36}$. For strength-dominated craters, growth stops at the end of the inertial regime, which occurs at $\tau=0.33\left(Y_{e f f} \rho U^{2}\right)^{-0.78}$, where $Y_{e f f}$ is the effective planetary crustal strength. The effective strength can be reduced from the ambient strength by fracturing and shear band melting (e.g. formation of pseudo-tachylites). In gravity-dominated craters, growth stops when the gravitational forces dominate over the inertial forces, which occurs at $\tau=0.92\left(\mathrm{ga}^{2}\right)^{-0.61}$. In the strength and gravity regimes, the maximum depth of penetration is $d_{p} / a=0.84\left(Y / \rho U^{2}\right)^{-0.28}$ and $d_{p} / a=$ $1.2\left(\mathrm{ga} / \mathrm{U}^{2}\right)^{-0.22}$, respectively. The transition from simple bowl-shaped craters to complex-shaped craters occurs when gravity starts to dominate over strength in the cratering process. The diameter for this transition to occur is given by $D_{t}=9.0 \mathrm{Y} / \mathrm{gg}$, and thus scales as $g^{-1}$ for planetary surfaces when strength is not strainrate dependent. This scaling result agrees with crater-shape data for the terrestrial planets [Chapman and McKinnon, 1986]. We have related some of the calculable, but nonobservable parameters which are of interest ( e.g. maximum depth of penetration, depth of excavation, and maximum crater lip height) to the crater diameter. For example, the maximum depth of penetration relative to the maximum crater diameter is 0.6 , for strength dominated craters, and 0.28 for gravity dominated craters. These values imply that impactors associated with the large basin impacts penetrated relatively deeply into the planet's surface. This significantly contrasts to earlier hypotheses in which it had been erroneously inferred from structural data that the relative transient crater depth of penetration decreased with increasing diameter. Similarly, the ratio of the maximum depth of excavation relative to the final crater diameter is a constant $\simeq 0.05$, for gravity dominated craters, and $=0.09$ for strength dominated craters. This result implies that for impact velocities less than $25 \mathrm{~km} / \mathrm{s}$, where significant vaporization begins to take place, the excavated material comes from a maximum depth which is less than 0.1 times the crater diameter. In the gravity dominated regime, we find that the apparent final crater diameter is approximately twice the transient crater diameter and that the inner ring diameter is less than the transient crater diameter.
\end{abstract}

\section{INTRODUCTION}

The recent decades of planetary observation and exploration have lead to the conclusion that the impact of solid bodies is one of the fundamental processes in the origin and evolution of the solar system. While the impact process is conceptually easy to visualize, the detailed quantitative description of the mechanics bave been difficult and illusive [e.g., Melosh, 1989]. The overall objective of this study is to obtain a quantitative description of the planetary cratering process over a broad range of conditions. Specifically the objectives are to establish quantitative scaling relationships for (1) the temporal evolution of the key measures of the crater geometry (depth and diameter), (2) the maximum depth of penetration and excavation, and lip height, and (3) the transition from simple to complex craters. We address these objectives over a broad range of planetary gravities, material strengths, and impactor sizes (from meters to those that formed the multiringed basins).

\section{Copyright 1993 by the American Geophysical Union.}

Paper number 93JE01330. 0148-0227/93/93JE-01330\$05.00

\section{APPROACH AND SCOPE}

We modeled the normal impact of spherical projectiles on a semi-infinite planet surface over a broad range of conditions using numerical techniques. We do not address the effect of a planet's atmosphere upon the cratering process in the present paper and refer readers to [Schultz and Gault, 1981; Melosh, 1989, chap. XI; Roddy et al., 1987; O'Keefe and Ahrens, 1988]. To calculate the impact-induced flow fields within the solid planets, we used the Eulerian-Lagrangian code developed by Thompson [1979]. The key equation of state parameters along with the mechanical parameters that were used in the code to represent a typical silicate impactor and planet are listed in Table 1. The constitutive model we employ is elasticplastic [Thompson, 1979]. Other models for material strength are being examined and will be reported upon in the future. We varied the mechanical properties as characterized by the yield strength. In all cases, the material properties of the impactor and planet were identical.

The impact parameters and variables are defined and listed in Table 1 along with the range over which they were varied. We nondimensionalized these using the formalism of Holsapple and Schmidt [1987]. The magnitudes of the 
TABLE 1. Scope of Parameters Studied for Impact of Silicate Projectile on a Planetary Half-Space

\begin{tabular}{|c|c|c|c|}
\hline Parameter & Symbol & Value Employed & Unit \\
\hline Impact velocity & $\boldsymbol{U}$ & 12 & $\mathbf{k m} / \mathbf{s}$ \\
\hline Planetary gravity & 8 & $0,1,10^{2}, 10^{4}, 10^{5}, 10^{6} \times g_{e}$ & $\operatorname{ge}\left(980 \mathrm{~cm} / \mathrm{s}^{2}\right)$ \\
\hline Density & $\mathbf{r}$ & 2.7 & $\mathrm{~g} / \mathrm{cm}^{3}$ \\
\hline Bulk modulus & $\boldsymbol{E}$ & $7.6 \times 10^{11}$ & $\mathrm{dyn} / \mathrm{cm}^{2}$ \\
\hline $\begin{array}{l}\text { Gruneisen } \\
\text { coefficient }\end{array}$ & $\mathbf{g}$ & 2.0 & - \\
\hline Melt enthalpy & $H_{m}$ & $1.1 \times 10^{10}$ & $\mathrm{ergs} / \mathrm{g}$ \\
\hline Yield strength & $\boldsymbol{Y}$ & $0,0.24,2.4,5.6,24,28,140,240,2400$ & kbar \\
\hline $\begin{array}{l}\text { Inverse Froúde } \\
\text { number }\end{array}$ & $g a / U^{2}$ & $\begin{array}{l}0.0,3.4 \times 10^{-7}, 3.4 \times 10^{-5}, 3.4 \times 10^{-3}, 3.4 \times 10^{-2} \\
3.4 \times 10^{-1}\end{array}$ & - \\
\hline $\begin{array}{l}\text { Inverse Cauchy } \\
\text { number }\end{array}$ & $Y / \pi U^{2}$ & $\begin{array}{l}0.0,6.2 \times 10^{-5}, 6.2 \times 10^{-4}, 1.4 \times 10^{-3}, 6.2 \times 10^{-3} \\
7.2 \times 10^{-3}, 3.6 \times 10^{-2}, 6.2 \times 10^{-2}, 6.2 \times 10^{-1}\end{array}$ & - \\
\hline Melt number & $H_{m} / U^{2} *$ & $4.65 \times 10^{-3}$ & \\
\hline
\end{tabular}

"Enthalpy for incipient melting.

three dimensionless parameters $g a / U^{2}, Y / \rho U^{2}$, and $H_{m} / U^{2}$ are measures of the dominant mechanisms controlling the cratering process. We varied these parameters over a range of conditions so as to determine when each of these was the dominant parameter that described the impact process.

The inverse Froúde number, $g a / U^{2}$, is a measure of the gravitational forces relative to the pressure forces. (This is the $\pi_{2}$ parameter identified by Schmidt [1980] without a factor of 3.22. This factor makes the expression numerically equal to the original definition by Schmidt [1977] in terms of impactor or explosive energy.) The inverse Cauchy number, $Y / \rho U^{2}$, is a measure of the planetary strength relative to the pressure forces; and the melt number, $H_{m} / U^{2}$, is a measure of the relative importance of melting. In determining the range of the inverse Froúde number, we had a choice of varying either the projectile radius or the gravitational acceleration; for ease of computation, we fixed the radius and varied the gravitational acceleration over six orders of magnitude. This is equivalent to varying the impactor radius from $5 \mathrm{~m}$ to $5000 \mathrm{~km}$. We restricted the impact velocities to 12 $\mathrm{km} / \mathrm{s}$ so as to not get into the impact regime where there are significant vaporization effects. This limits the validity of the scaling laws to velocities less than $30 \mathrm{~km} / \mathrm{s}$ for typical silicates. We have studied the effects of high speed impacts $(>30 \mathrm{~km} / \mathrm{s})$ and will report in detail on these elsewhere (see preliminary work in $O^{\prime}$ Keefe and Ahrens [1989]).

The results of the computations represent a very large amount of computer output with over $\sim 10^{4}$ variables being calculated for each time step. In this paper we will report on the geometrical measures of the cratering process such as the depth, diameter and lip height. The depth is defined as the distance from the initial planetary surface and the planetary surface at a given time at the centerline of the impact; the diameter is defined as the distance between the interface between the impactor and planetary surface at the initial planetary surface height; the lip height is measured relative to the original planetary surface level (e.g., see Figure 1). The dimensionless depth and diameter histories are summarized in Table 2 . In addition, we have included a series of detailed flow field plots; these represent the development of a simple bowl-shaped crater (Figure 1) and a complex crater exhibiting central peak and ring formation (Figures 2 and 3). To determine the displacement of planetary material during the cratering process, we placed massless tracer particles at various positions and computed their trajectories which are also shown in the right sides of Figures 1 and 2 . In addition, we connected the tracer particles both vertically and horizontally so as to illustrate the deformations that occur within the surface layers. These results can be compared to field measurements of the deformations of the layering sequences and can also be compared to laboratory scale experiments. The results for a complex crater are shown in Figure 3.

\section{Crater Scaling Regimes}

The cratering process can be described in terms of at least four temporal regimes; penetration, inertial, terminal and relaxation. Schmidt and Holsapple called these first three regimes the early stage, intermediate and late. The numerical approach taken here can be used to describe the first three; the fourth, the relaxation regime is the result of long term equilibration and requires a different analytical approach such as viscous relaxation methods [e.g., Melosh, 1989, chap. 8]. In the following section we will describe the first three regimes and determine the numerical values of the parameters in the Holsapple and Schmidt [1987] scaling laws and compare these with values measured experimentally by Schmidt and Housen [1987]. We will describe the depth, diameter, and other geometrical measures in that order. The scaling laws and the numerical values of the parameters derived from the present calculations are listed in Table 3. 

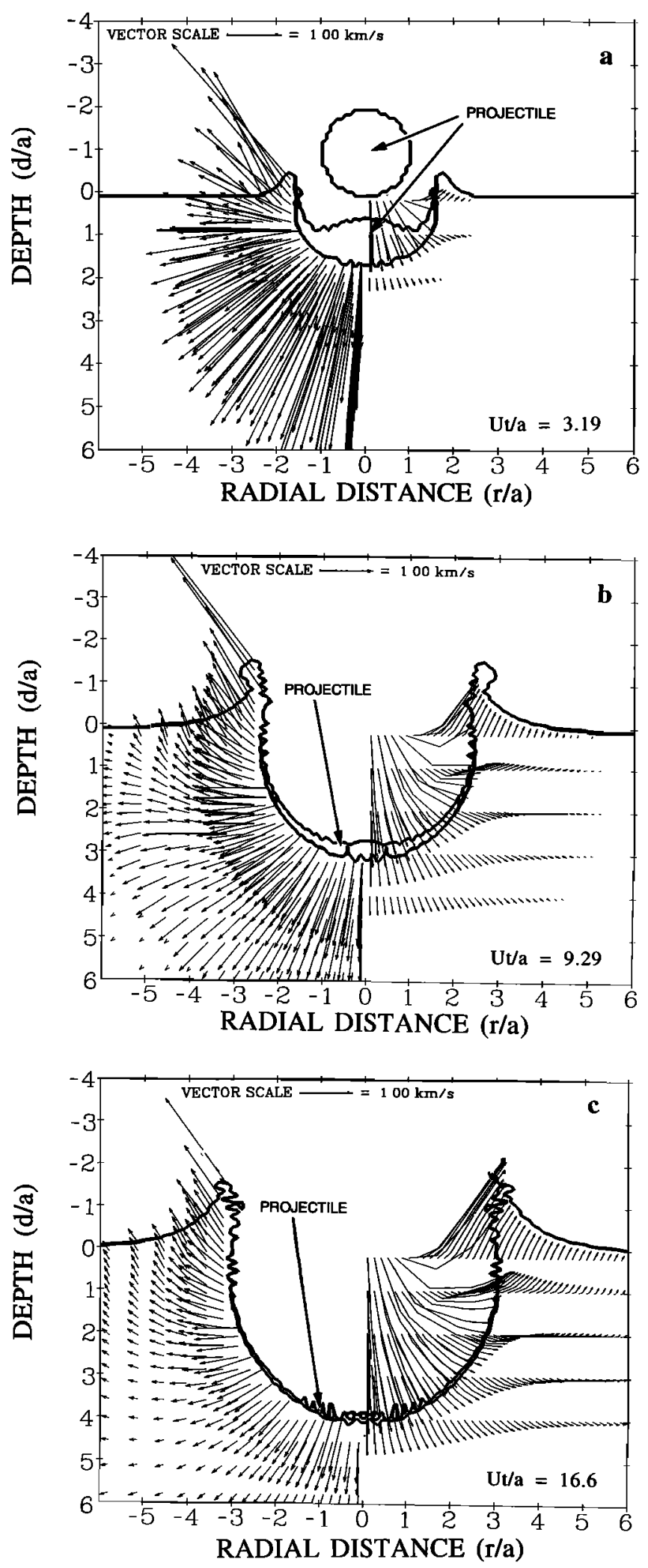

Fig. 1. Simple bowl shaped crater. Velocity and deformation history fields for the impact of a silicate projectile on a silicate half-space.The depth and radial distance are nondimensionalized by the impactor radius, a. The flow field is in the strenglh- dominated regime with $g a / U^{2}=3.4 \mathrm{x}$ $10^{-7}$ and $Y / \rho U^{2}=1.4 \times 10^{-3}$. The velocity field is shown on the left side with the scale shown at the top. On the right-hand side are the trajectories of tracer particles placed at various deplhs. Arrows on tracer trajectories indicate the particle position at the specified time and direction of motion. The panels represent dimensionless times of 3.19, 9.29, and 16.6. This is representative of a simple bowl-shaped crater.

\section{Penetration Regime}

The penetration regime is characterized by the transfer of the kinetic energy of the projectile to the planetary surface. In the case where the impactor and planetary surface have similar properties, about half of the impactor kinetic energy is transferred to the planet during the penetration regime [O'Keefe and Ahrens, 1977]. During this time, the impactor drives a strong shock wave into the planet and also deforms and lines the growing crater cavity wall (Figure 1a). The material properties of the impactor and the planet and the planet's gravitational acceleration are not important during this time, except for relative differences in density between the impactor and the planet [Holsapple and Schmidt, 1987].

Depth (d)

The crater depth grows linearly with time in the penetration regime. Shown in Figure 4 is a plot of the evolution of the interface between the impactor and the planet at the centerline of impact for a wide range of impact conditions. Note that at early times, all the cases fall on a single line independent of strength or gravitational acceleration. This linear growth is expected during the early times when the shock at the interface is nearly planar and is predicted from planar shock wave theory. The $\operatorname{depth}(d) /$ impactor $\operatorname{radius}(a)$ evolves as

$$
\frac{d}{a}=\frac{1}{2}(U t / a)
$$

where $U$ is the impact velocity. The factor of one-half comes from the fact that upon impact of bodies of like impedances, the interface velocity is one-half the impactor velocity.

The depth grows linearly with time until it enters the inertial regime This was predicted by Holsapple and Schmidt [1987] to occur at dimensionless time, $\tau$, of 5.1 for like impacts and this is substantiated in Figure 4.

\section{Diameter (D)}

The diameter does not have a simple growth law during the penetration regime. The impact of a near spherical impactor will produce small amounts of jetting and vaporization near the initial point of impact even at low velocities; the magnitude depends sensitively on the details of the impactor-planetary surface geometry [Kieffer, 1976]. The penetration regime for diameter ends when the maximum lateral extent of the impactor penetrates the surface which, from geometrical considerations for a spherical impactor, occurs at dimensionless time of

$$
\tau=\frac{U t}{a}=1
$$

The cratering process during the penetration regime scales as the energy of impact. This is because the ejecta plume is not significant and all of the energy is in the crater cavity. This also implies that phenomena that are dominated by processes during the penetration regime will scale as the energy of impact [Bjorkman and Holsapple, 
TABLE 2. Normalized Crater Diameter and Depth Versus $t$ for Different Values of ga/ ${ }^{2}$, and $Y / r U^{2}$

\begin{tabular}{|c|c|c|c|}
\hline$\frac{\mathbf{g a}}{\mathbf{U}^{\mathbf{2}}}$ & $\frac{\mathrm{Y}}{\mathrm{IU}^{2}}$ & $\frac{\mathrm{D}}{\mathrm{a}}$ & $\frac{d}{a}$ \\
\hline $0(\mathrm{U}=10 \mathrm{~km} / \mathrm{s})^{*}$ & 0.0 & & $\begin{array}{l}0.2 \\
0.8 \\
2.0 \\
3.0 \\
4.0\end{array}$ \\
\hline $0(\mathrm{U}=50 \mathrm{~km} / \mathrm{s})^{*}$ & 0.0 & & $\begin{array}{l}0.4 \\
1.0 \\
2.0 \\
2.6 \\
4.0 \\
5.2\end{array}$ \\
\hline $0(\mathrm{U}=100 \mathrm{~km} / \mathrm{s})^{*}$ & 0.0 & & $\begin{array}{l}0.2 \\
1.0 \\
2.0 \\
2.6 \\
4.0 \\
5.0 \\
6.0 \\
7.0 \\
8.0 \\
9.5\end{array}$ \\
\hline $3.4 \times 10^{-7}$ & $1.4 \times 10^{-3}$ & $\begin{array}{l}3.40 \\
5.10 \\
5.70 \\
6.50 \\
6.60 \\
7.40 \\
7.80 \\
8.80 \\
9.00 \\
9.00 \\
9.00 \\
9.00\end{array}$ & $\begin{array}{l}1.65 \\
3.00 \\
3.35 \\
3.75 \\
3.95 \\
4.40 \\
4.75 \\
5.15 \\
5.20 \\
5.10 \\
5.10 \\
5.00\end{array}$ \\
\hline $3.4 \times 10^{-7}$ & $7.2 \times 10^{-3}$ & $\begin{array}{l}3.50 \\
4.50 \\
5.00 \\
5.50 \\
5.70 \\
6.00 \\
6.00 \\
5.70 \\
5.60 \\
5.40\end{array}$ & $\begin{array}{l}1.70 \\
2.70 \\
3.00 \\
3.50 \\
3.70 \\
3.75 \\
3.60 \\
3.40 \\
3.20 \\
2.80\end{array}$ \\
\hline $3.4 \times 10^{-7}$ & $3.6 \times 10^{-2}$ & $\begin{array}{l}3.30 \\
4.35 \\
4.60 \\
4.60 \\
4.50 \\
4.20 \\
4.00 \\
3.80 \\
2.10 \\
1.00\end{array}$ & $\begin{array}{l}1.75 \\
2.90 \\
3.10 \\
2.95 \\
2.70 \\
2.50 \\
2.45 \\
2.30 \\
2.20 \\
1.50\end{array}$ \\
\hline $3.4 \times 10^{-5}$ & 0.0 & & $\begin{array}{l}0.37 \\
1.16 \\
1.67 \\
2.17 \\
2.60 \\
3.02 \\
3.53 \\
4.05 \\
4.42\end{array}$ \\
\hline
\end{tabular}


TABLE 2. (continued)

\begin{tabular}{|c|c|c|c|c|}
\hline$\frac{g a}{U^{2}}$ & $\frac{Y}{r U^{2}}$ & $\frac{\mathrm{D}}{\mathbf{a}}$ & $\frac{d}{a}$ & $\mathbf{t}$ \\
\hline $3.4 \times 10^{-3}$ & 0.0 & & $\begin{array}{l}0.40 \\
1.20 \\
1.90 \\
2.63 \\
3.25 \\
4.10 \\
4.10 \\
4.20 \\
3.45 \\
3.10 \\
1.40\end{array}$ & $\begin{array}{r}0.825 \\
2.360 \\
4.480 \\
7.890 \\
13.279 \\
22.770 \\
27.600 \\
32.000 \\
36.800 \\
41.200 \\
50.600\end{array}$ \\
\hline $3.4 \times 10^{-3}$ & $6.2 \times 10^{-5}$ & & $\begin{array}{l}0.1 \\
0.40 \\
0.75 \\
1.20 \\
1.50 \\
1.60 \\
1.70 \\
2.00 \\
2.30 \\
2.70 \\
2.50 \\
2.90 \\
3.20 \\
3.60 \\
3.80 \\
3.90 \\
3.65 \\
3.00 \\
2.00\end{array}$ & \begin{tabular}{r}
\multicolumn{1}{l}{0.2} \\
0.854 \\
1.550 \\
2.370 \\
3.090 \\
3.370 \\
3.640 \\
5.200 \\
6.070 \\
8.350 \\
7.200 \\
9.660 \\
12.690 \\
17.100 \\
21.900 \\
27.800 \\
35.400 \\
42.600 \\
50.400
\end{tabular} \\
\hline $3.4 \times 10^{-3}$ & $6.2 \times 10^{-4}$ & & $\begin{array}{l}0.50 \\
1.10 \\
1.70 \\
2.15\end{array}$ & $\begin{array}{l}0.907 \\
2.052 \\
3.280 \\
4.776\end{array}$ \\
\hline $3.4 \times 10^{-3}$ & $6.2 \times 10^{-3}$ & & $\begin{array}{l}0.50 \\
1.22 \\
1.85 \\
2.35 \\
2.55 \\
3.00 \\
3.35 \\
3.30 \\
3.00 \\
2.50 \\
2.20 \\
1.85\end{array}$ & $\begin{array}{r}0.934 \\
2.324 \\
3.710 \\
5.560 \\
7.040 \\
10.150 \\
15.090 \\
20.020 \\
27.600 \\
35.470 \\
43.200 \\
50.850\end{array}$ \\
\hline $3.4 \times 10^{-2}$ & $6.2 \times 10^{-5}$ & $\begin{array}{l}2.00 \\
2.75 \\
3.30 \\
3.55 \\
3.85 \\
4.10 \\
4.25 \\
4.85 \\
6.60 \\
8.35 \\
8.60 \\
8.10 \\
8.20 \\
8.60 \\
8.60\end{array}$ & $\begin{array}{l}0.400 \\
1.100 \\
1.700 \\
2.000 \\
2.350 \\
2.450 \\
2.450 \\
2.200 \\
1.950 \\
1.550 \\
1.150 \\
0.650 \\
0.500 \\
0.350 \\
0.300\end{array}$ & $\begin{array}{r}0.828 \\
2.120 \\
3.380 \\
4.580 \\
6.020 \\
7.990 \\
10.200 \\
13.700 \\
17.600 \\
21.300 \\
24.900 \\
28.500 \\
31.400 \\
34.000 \\
34.800\end{array}$ \\
\hline
\end{tabular}


TABLE 2. (continued)

\begin{tabular}{|c|c|c|c|c|}
\hline$\frac{\mathbf{g a}}{\mathrm{U}^{2}}$ & $\frac{\mathbf{Y}}{\mathrm{rU}^{2}}$ & $\frac{\mathrm{D}}{\mathrm{a}}$ & $\frac{d}{a}$ & $\mathbf{t}$ \\
\hline $3.4 \times 10^{-2}$ & $6.2 \times 10^{-4}$ & $\begin{array}{r}5.20 \\
9.15 \\
9.10 \\
9.10 \\
9.10 \\
9.10 \\
10.00\end{array}$ & $\begin{array}{l}2.20 \\
1.55 \\
0.80 \\
0.40 \\
0.35 \\
0.20 \\
0.30 \\
0.40\end{array}$ & $\begin{array}{l}13.75 \\
21.30 \\
28.56 \\
34.10 \\
34.80 \\
38.92 \\
44.88 \\
49.92\end{array}$ \\
\hline $3.4 \times 10^{-2}$ & $6.2 \times 10^{-3}$ & $\begin{array}{l}2.70 \\
2.95 \\
4.40 \\
4.60 \\
5.05 \\
5.55 \\
6.50 \\
7.50 \\
8.20 \\
9.40\end{array}$ & $\begin{array}{l}1.35 \\
2.15 \\
2.35 \\
2.35 \\
2.15 \\
2.00 \\
1.85 \\
1.60 \\
1.40 \\
0.80\end{array}$ & $\begin{array}{r}2.92 \\
5.60 \\
8.32 \\
10.20 \\
12.80 \\
14.40 \\
16.20 \\
17.90 \\
19.60 \\
23.80\end{array}$ \\
\hline $3.4 \times 10^{-2}$ & $6.2 \times 10^{-2}$ & $\begin{array}{l}4.85 \\
5.10 \\
5.30 \\
7.50 \\
8.10\end{array}$ & $\begin{array}{l}2.05 \\
1.85 \\
1.65 \\
0.85 \\
0.10\end{array}$ & $\begin{array}{l}13.89 \\
15.84 \\
17.76 \\
24.96 \\
32.64\end{array}$ \\
\hline $3.4 \times 10^{-2}$ & $6.2 \times 10^{-1}$ & $\begin{array}{l}3.00 \\
3.35 \\
3.80 \\
4.00 \\
3.95 \\
3.95 \\
4.15 \\
4.20\end{array}$ & $\begin{array}{l}1.35 \\
1.75 \\
2.25 \\
2.40 \\
2.35 \\
2.35 \\
2.20 \\
1.95\end{array}$ & $\begin{array}{r}2.61 \\
3.69 \\
6.45 \\
8.64 \\
9.98 \\
10.50 \\
12.60 \\
18.00\end{array}$ \\
\hline $3.4 \times 10^{-2}$ & 6.2 & $\begin{array}{l}3.20 \\
3.80 \\
4.05 \\
4.15 \\
4.20 \\
4.05\end{array}$ & $\begin{array}{l}1.45 \\
2.40 \\
2.40 \\
2.35 \\
2.25 \\
2.00\end{array}$ & $\begin{array}{r}2.660 \\
6.766 \\
9.040 \\
11.400 \\
12.900 \\
16.300\end{array}$ \\
\hline 0.34 & 0.0 & $\begin{array}{l}2.1 \\
2.6 \\
2.6 \\
2.4 \\
2.3 \\
4.0 \\
4.3 \\
4.5 \\
5.0 \\
5.2 \\
5.3 \\
5.8\end{array}$ & $\begin{array}{l}0.40 \\
1.10 \\
1.50 \\
1.50 \\
1.50 \\
1.45 \\
1.40 \\
1.35 \\
1.30 \\
1.30 \\
1.25 \\
1.20\end{array}$ & $\begin{array}{l}0.765 \\
2.010 \\
3.500 \\
3.900 \\
4.080 \\
4.180 \\
4.480 \\
4.750 \\
5.220 \\
5.350 \\
5.678 \\
7.050\end{array}$ \\
\hline 0.341 & $6.2 \times 10^{-5}$ & $\begin{array}{l}2.20 \\
2.45 \\
2.7 \\
2.8 \\
2.9 \\
2.7\end{array}$ & $\begin{array}{l}0.38 \\
0.68 \\
1.15 \\
1.30 \\
1.35 \\
1.43\end{array}$ & $\begin{array}{l}0.761 \\
1.290 \\
2.180 \\
2.490 \\
2.950 \\
3.400\end{array}$ \\
\hline
\end{tabular}

-Time history taken from Holsapple and Schmidt [1987].

The impact velocity $U$ was $12 \mathrm{~km} / \mathrm{s}$ except where indicated. 

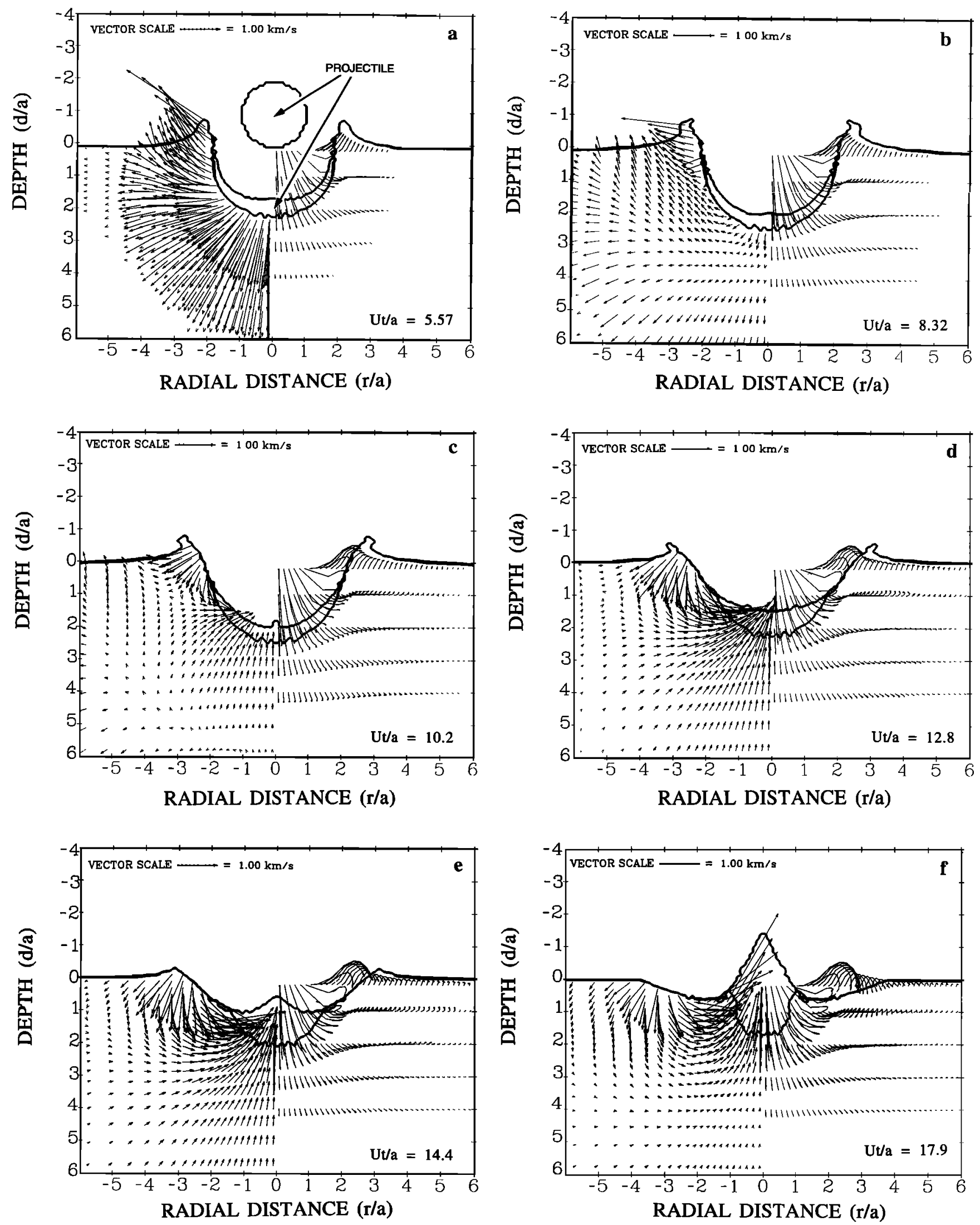

Fig. 2. Complex crater. Velocity and deformation history fields for the impact of a silicate projectile on a silicate half-space. The depth and radial distance are nondimensionalized by the impactor radius, $a$. The flow field is gravity dominated at late times with $\mathrm{ga} / U^{2}=3.4 \times 10^{-2}$ and $Y / \rho U^{2}=6.2 \times 10^{-3}$. The velocity field is shown on the left side with the scale at the top. On the right side are the trajectories of tracer particles placed at various depths. The panels represent dimensionless times of 5.57, 8.32, 10.2, 12.8, 14.4, 17.9, and 19.7. This is representative of a complex crater. 


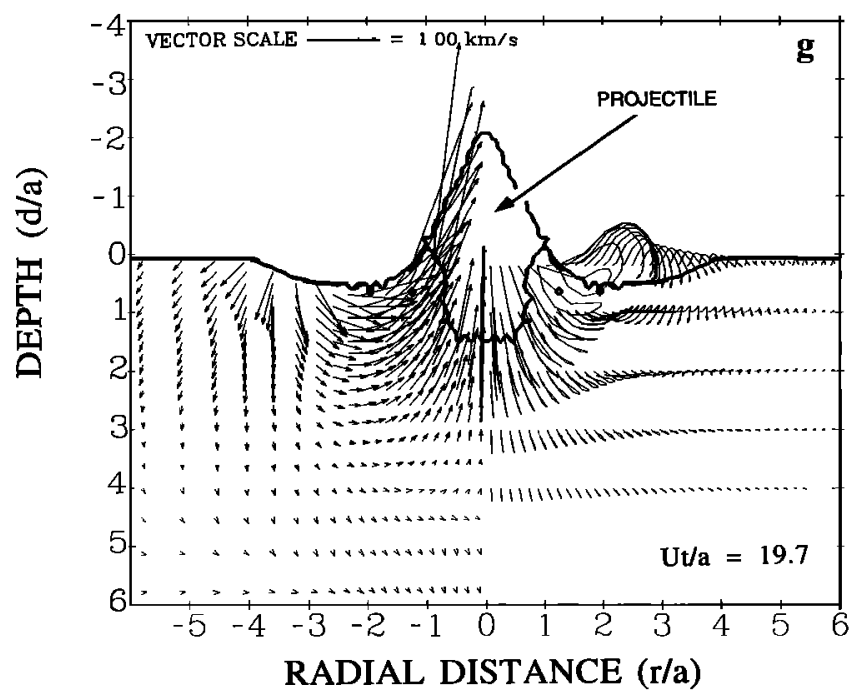

Fig. 2 (continued)

1987; O'Keefe and Ahrens, 1993]. Examples of this are the amount of melting and vaporization.

\section{Shape}

The shape of the transient cavity changes rapidly during the penetration regime. The depth grows more rapidly than the diameter during the penetration time, and the scaling of the evolution of the diameter to depth is given from the ratio of equations (6) and (1)

$$
\frac{D}{d}=4.0\left(\frac{U t}{a}\right)^{-0.64} \quad 1<\left(\frac{U t}{a}\right)<5.1
$$

\section{INERTIAL, REGIME}

The inertial regime is characterized by the quasihemispherical expansion of the crater cavity. In this regime, the geometry of the cavity does not change, and the projectile is deformed into a thin hemispherical shell that lines the transient crater cavity. The strong shock that was attached to the projectile during the penetration time has now propagated away from the the cavity region [Bjork, 1961; O'Keefe and Ahrens, 1977]. Bjork called this regime the detached shock regime. As in the penetration regime, the material strength properties and gravity do not play a role in the evolution, however, the termination of this regime occurs when either strength or gravitational effects arrest the crater growth. Examples of the flow fields and deformation in the inertial regime are given in Figures $1 b, 1 c, 2 a, 2 b$, and $3 b$.

\section{Depth (d)}

The depth grows as a simple power law in the inertial regime and it is independent of either strength or gravitational acceleration (see Figure 4). A similar result for the depth was found by Holsapple and Schmidt [1987, Figure 5] for a variety of impact conditions. Equation (4) is obtained by fitting the depth versus time curve (Figure 4) in the inertial regime. The scaling law is given by

$$
\frac{d}{a}=1.3(U t / a)^{0.36}
$$

The magnitude of the inertial regime exponent $(S=0.36)$ is related to the coupling exponent of Holsapple and Schmidt [1987] by

$$
\mu=S /(1-S)
$$

Equation 5 gives $\mu=0.56$, which is consistent with the range of values found by Holsapple and Schmidt [1987] and Schmidt and Housen [1987] for a range of impact conditions. In the following sections all of the scaling laws will have exponents that are functions of $\mu$ and thus are related to the exponent in the inertial regime.

\section{Diameter (D)}

The diameter also grows as a simple power law in the inertial regime (e.g. Figure 5). The scaling for the diameter from a fit to the results in Figure 5 is given by

$$
\frac{D}{a}=2.0(U t / a)^{0.36}
$$

Note that the exponent is the same for both the depth and diameter.

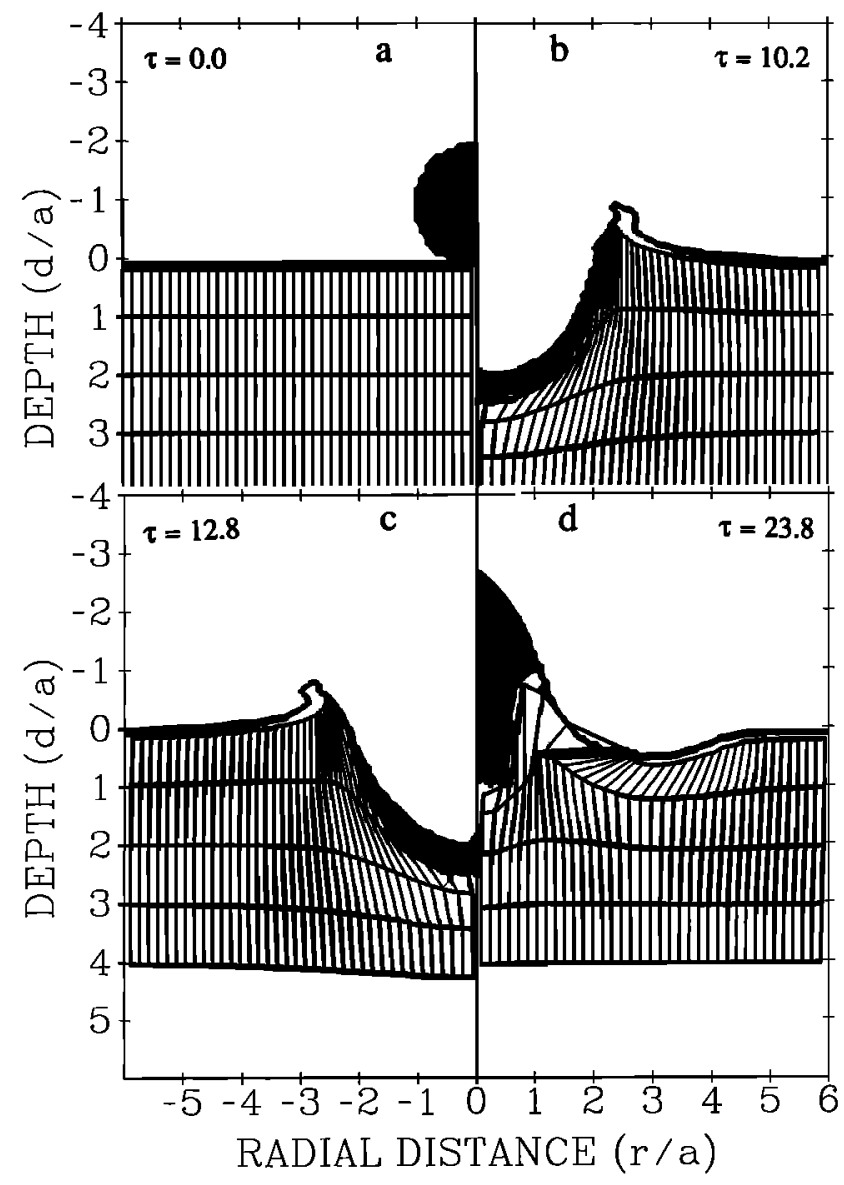

Fig. 3. Complex crater. The massless tracer particles have been connected both vertically and horizontally so as to show the deformations that would be observed in the stratigraphy during the impact process. The impact parameters are the same as Figure $2,8 \mathrm{ga} / U^{2}=3.4 \times 10^{-2}$ and $Y / \mathrm{p} U^{2}=6.2 \times 10^{-3}(a)$ prior to impact, $(b)$ deformations near the time of maximum depth of penetration, (c) growth of diameter as a result of the collapse and propagation of the transient crater lip, $(d)$ the deformations driving rebound and development of inner and outer rings. 
TABLE 3. Summary of Crater Scaling Formula

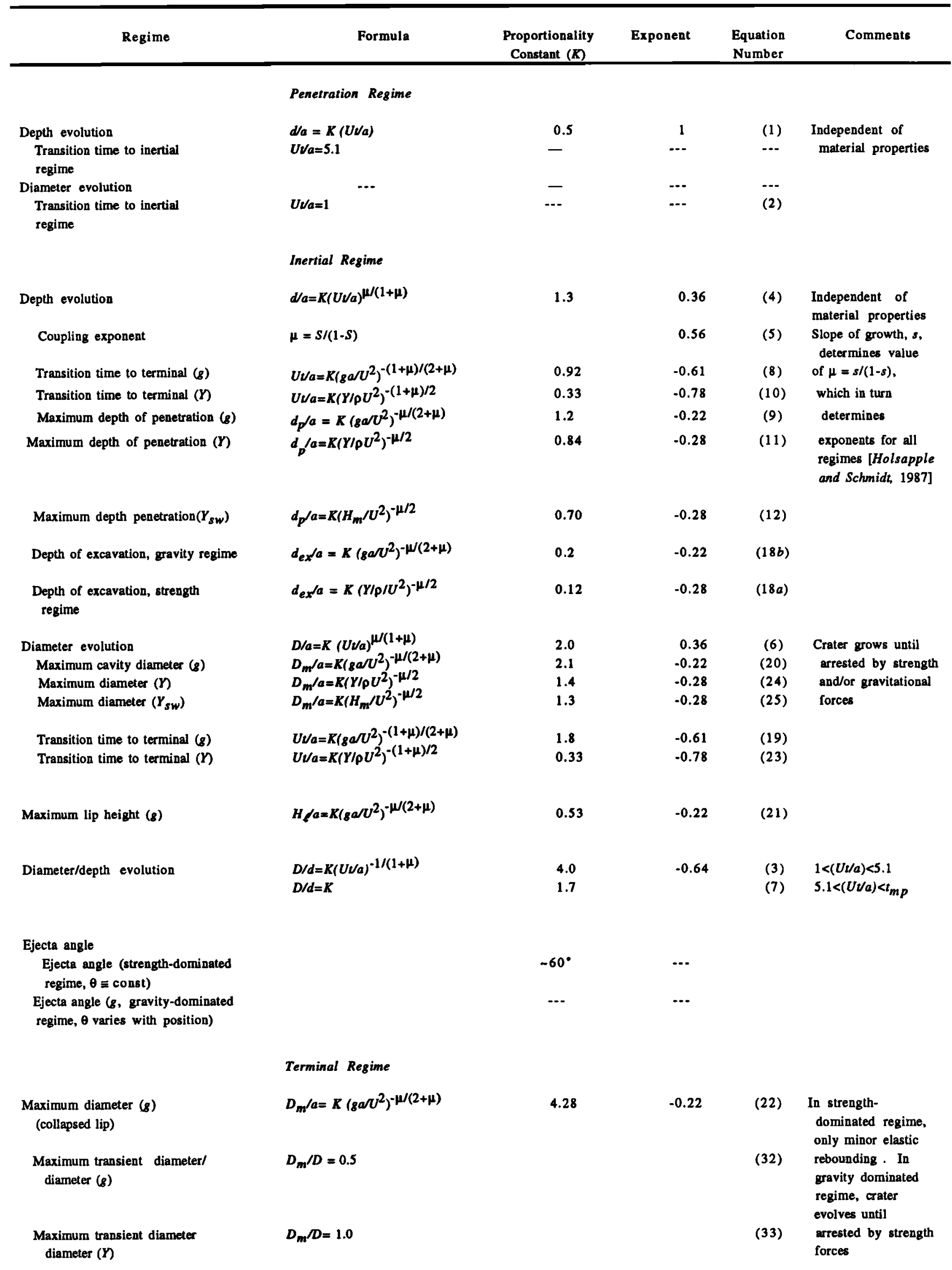


TABLE 3. (continued)

\begin{tabular}{|c|c|c|c|c|c|}
\hline Regime & Formula & $\begin{array}{c}\text { Proportionality } \\
\text { Constant }(K)\end{array}$ & Exponent & $\begin{array}{l}\text { Equation } \\
\text { Number }\end{array}$ & Comments \\
\hline Maximum lip height/diameter & $H_{\ell} / d=0.12$ & & & (34) & \\
\hline Depth of penetration/diameter $(g)$ & $d_{p} / D=0.28$ & & & (36) & \\
\hline Depth of penetration/diameter $(Y)$ & $d_{p} / D=0.6$ & & & (37) & \\
\hline Depth of excavation/diameter (g) & $d_{e x} / D=0.05$ & & & (38) & \\
\hline Depth of excavation/diameter $(Y)$ & $d_{e x} / D=0.085$ & & & (39) & \\
\hline $\begin{array}{l}\text { Transition diameter from } \\
\text { simple to complex craters }\end{array}$ & $D_{t}=K(Y / P g)$ & 9.0 & - & (28) & \\
\hline
\end{tabular}

Explanation of symbols:

$(g)$, regime indicated is gravity dominated; $(Y)$, regime indicated is strength dominated; and $Y_{s w}$, regime indicated is shock wave degraded strength dominated.

\section{Shape}

The shape is similar throughout the duration of the inertial regime. The ratio of the diameter to depth is

$$
\frac{D}{d}=1.67 \quad 1<(U t / a)<t_{m p}
$$

where $t_{m p}$ is the time of maximum penetration. This time is specified in (8) below.

\section{Ejecta}

The angle of ejecta, $\theta$, is defined as the angle that material is ejected as measured from the undisturbed surface (horizontal). In the case of strength dominated craters, $\theta$, is constant and does not vary with radial position on the surface (e.g., Figure 1c). In this case $\theta-60^{\circ}$. This constant angle for strength dominated craters was found also by Housen et al. [1983]. In the case of gravity dominated craters, $\theta$, varies with radial position or, equivalently, the time of ejection (see Figures $2 a$ and $2 b$ ). Also, $\theta$ increases with radial position and is consistent with the water impact results of Gault and Sonett [1982].

\section{Terminal Regime}

The growth in inertial regime is arrested by strength and gravitational forces, and this marks the beginning of the terminal regime. In the case of simple craters (Figure 1), the final shape of the crater is reached at the end of the

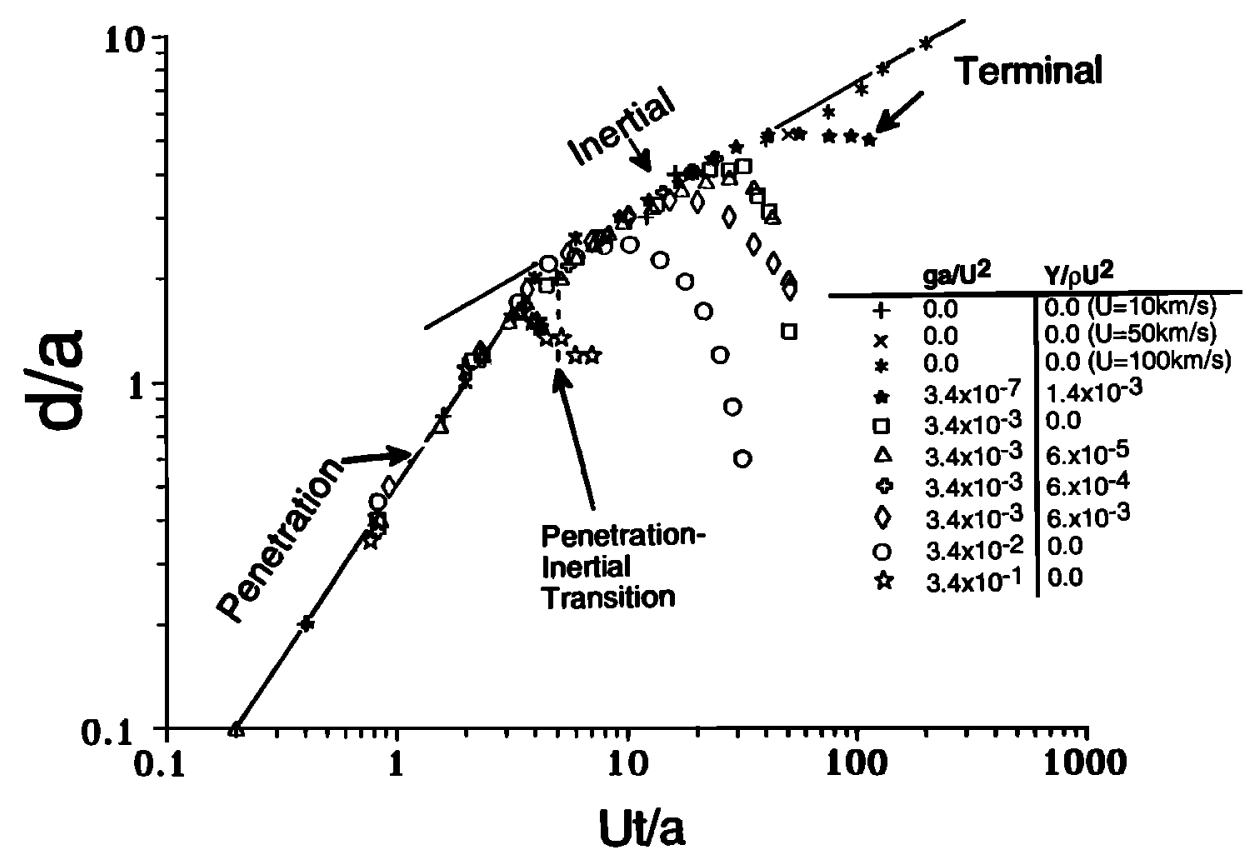

Fig. 4. Dimensionless depth $(d / a)$ versus dimensionless time $(U t / a)$ for the penetration, inertial and terminal regimes for different values of $\mathrm{g} a / U^{2}$ and $Y / \rho U^{2}$. 


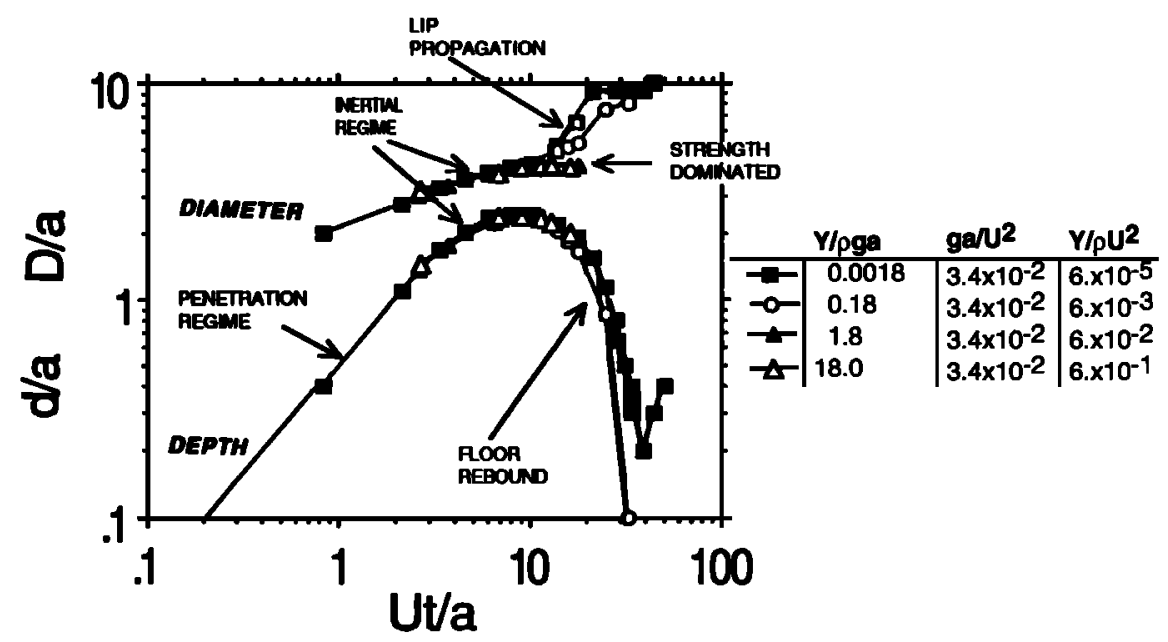

Fig. 5. Temporal evolution of the dimensionless depth of penetration $(d / a)$ and crater diameter $(D / a)$ for a fixed value of inverse Froude number $\left(\mathrm{g} a / U^{2}=0.034\right)$ and various values of $\mathrm{Y} / \mathrm{pga}$.

inertial regime with the exception of the collapsing and folding over of the crater lip and minor elastic rebounding. In the case of complex craters, the end of the inertial regime marks the beginning of a series of complex motions (e.g. Figures $2 c$ to $2 g, 3 c$ and $3 d$ ). These motions include the rebounding of the crater floor and the formation of a central peak and the collapse and propagation of the crater lip to form crater rings.

Depth (d)

The beginning of the terminal regime for crater depth occurs at the time of maximum penetration, $t_{m p}$. This time, in the case where the gravitational forces dominate over the strength forces, is given by

$$
\frac{U t_{m p}}{a}=0.92\left(\frac{g a}{U^{2}}\right)^{-0.61}
$$

where the exponent magnitude is given by $(1+\mu) /(2+\mu)$. The proportionality constant is obtained by fitting the results shown in Figure 4. Note that the proportionality constant $(0.92)$ in equation 8 , is intermediate in value between 0.79 for wet sand and 1.1 for water, and the exponent $\mu$ is 0.56 which compares favorably with the exponent 0.55 found for both water and wet sand [Schmidt and Housen, 1987]. The flow and deformation field near the time of maximum penetration are shown in Figures $1 c$, $2 b$, and $3 b$.

The maximum in the depth of penetration in the gravity dominated case is a function of the inverse Froúde number and is obtained from Figure 6. The associated scaling law is given by

$$
\frac{d_{p}}{a}=1.2\left(\frac{g a}{U^{2}}\right)^{-0.22}
$$

where the magnitude of the exponent is given by $\mu /(2+\mu)$ and the proportionality constant is obtained from the results shown in Figure 6.

The time of maximum penetration $t_{m p}$, and the beginning of the terminal regime, in the case where strength forces are dominant is given by

$$
\frac{U t_{m p}}{a}=0.33\left(\frac{Y}{\rho U^{2}}\right)^{-0.78}
$$

where the magnitude of the exponent is $(1+\mu) / 2$ Here $Y$ is equal to twice the maximum shear strength. For the crusts of typical cratered planetary surfaces, we expect $Y$ will be significantly lower in magnitude from data from tests on undamaged rock on account of shock-induced cracking [e.g., Simmons et al., 1973; Ahrens and Rubin, 1993]. The temporal evolution in the strength dominated regime is shown in Figure 7 from which (10) is derived by fitting the peaks of $d / a$ versus $U t / a$ relations.

The maximum depth of penetration in the strength dominated case is a function of the inverse Cauchy number and is shown in Figure 8. The depth follows a power law with an expected slope given by $-\mu / 2=-0.28$ until the inverse Cauchy number approaches $\sim 10^{-2}$. This change in scaling is a result of increased importance of shock weakening of the planetary material in the cratering process. Weakening is a result of the irreversible work

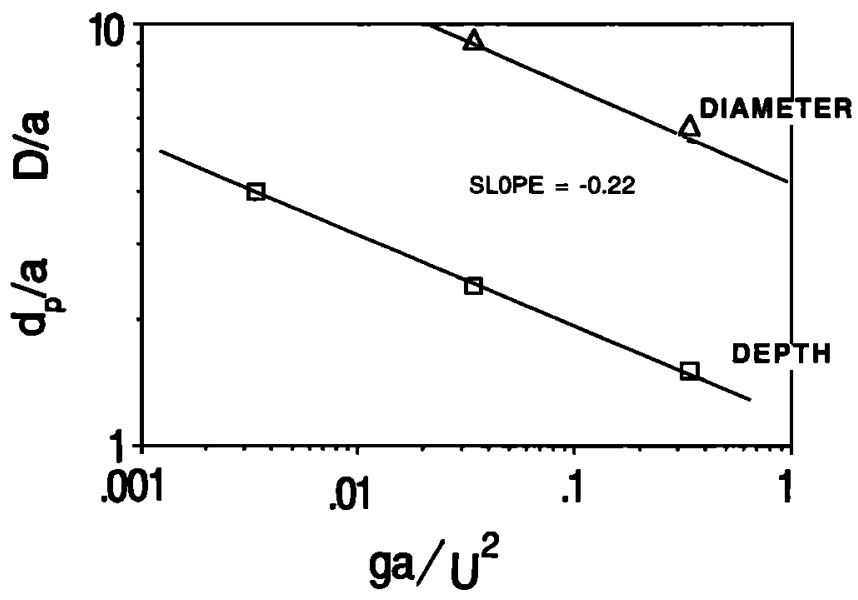

Fig. 6. Dimensionless maximum depth of penetration $(d p / a)$ and maximum diameter $(D / a)$ as a function of inverse Frotede number $\left(g a / U^{2}\right)$. Slope corresponds to $-\mu /(2+\mu)$ for gravity scaling. Symbols are results for different calculations. 


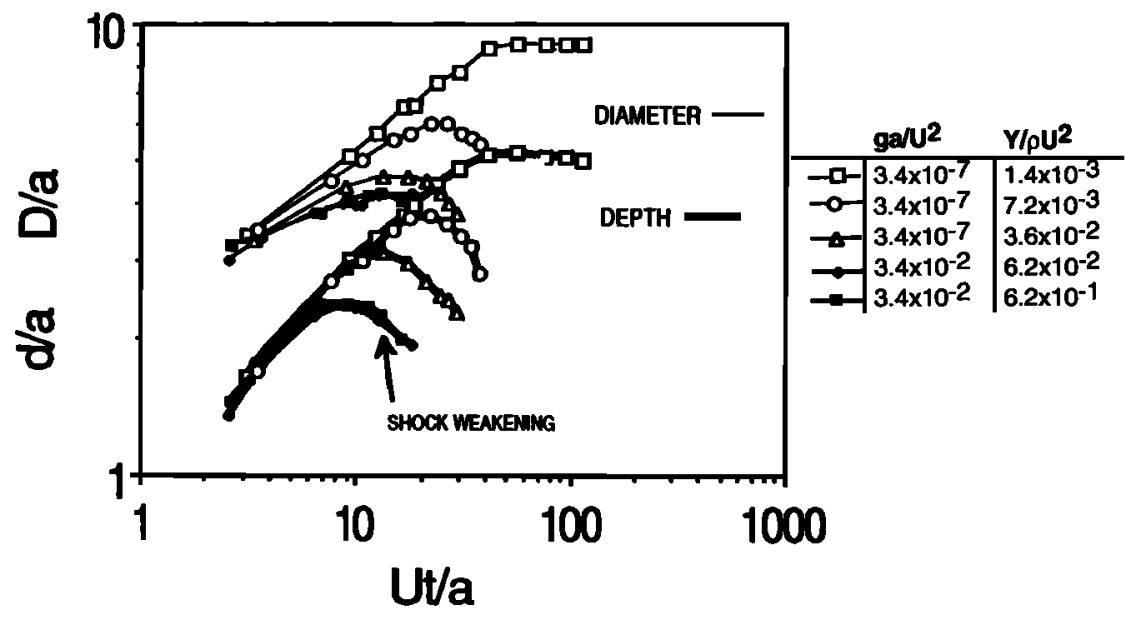

Fig. 7. Temporal evolution of dimensionless depth $(d / a)$ and diameter $(D / a)$ in the strength-dominated regime.

done in by the shock and subsequent unloading process and as a result of the postshock deformation of the rock surrounding the crater. The irreversible work will result from both thermal shock heating and mechanical fracturing effects and is more intense close to the impact point where the high shock stresses are experienced. The importance of shock weakening depends upon the planetary strength, thermal state, and impact velocity. In the case of weak planetary crusts, and low impact velocities, the volume of the final crater is much greater than the shock weakened region and the effect is minor. However in the case of very strong initial crustal strengths, high surface temperatures or high $(>30 \mathrm{~km} / \mathrm{s})$ impact velocities, the extent of the shock weakening is comparable to the crater size. This condition applies to craters in the $\mathrm{mm}$ diameter or smaller range on hard rock. In these cases, the shock weakening effect can dominate late stage crater growth and the entire size and shape of the final crater.

Shock weakening was modeled as thermal weakening and did not explicitly account for fracturing, which is currently an area of active interest [e.g., Ahrens and Rubin, 1993; Asphaug et al., 1991; Housen and Holsapple, 1990] and the present work should be extended to include that effect. Our code computed the temperature field at each time step. The

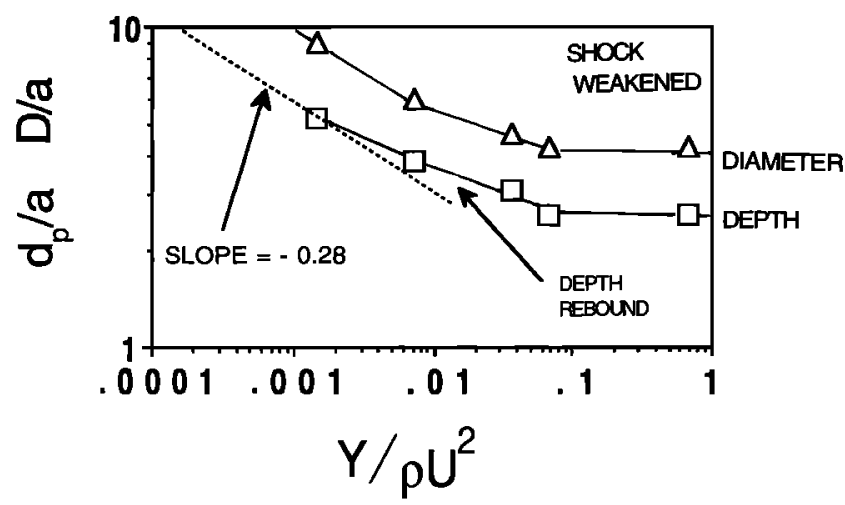

Fig. 8. Dimensionless maximum depth of penetration $\left(d_{p} / a\right)$ and diameter $(D / a)$ as a function of inverse Cauchy number $\left(Y / \rho U^{2}\right)$. The slope for small values of the inverse Cauchy number is $-0.28=-\mu / 2$ and corresponds to strength scaling. The change in slope is a result of melting and shock weakening. Symbols represent different calculations for a fixed value of Frocide number $\left(\mathrm{g} a / U^{2}=0.034\right)$ and melt number $\left(H_{m} / U^{2}=4.65 \times\right.$ $10^{-3}$ ). local temperature was used to compute the degree of thermal weakening by reducing the local strength in proportion to the difference between temperature and the melt temperature where the strength vanishes. The results of the code runs are plotted in Figure 8 for both depth of penetration and diameter.

In the strength dominated case where shock weakening is negligible, the scaling is given by

$$
\frac{d_{p}}{a}=0.84\left(\frac{Y}{\rho U^{2}}\right)^{-0.28}
$$

which is derived from Figure 8.

In the shock weakened case, the depth is independent of the magnitude of the yield strength and is determined by the amount of material that is thermally weakened and is given by

$$
\frac{d_{p}}{a}=0.70\left(\frac{H_{m}}{U^{2}}\right)^{-0.28}
$$

where $H_{m}$ is the enthalpy required for incipient melting, which in the case of thermal weakening is equal to the enthalpy for incipient melting as referenced to the planets' ambient surface condition. To assess the effect of shock fracturing, $H_{m}$ can be replaced by $H_{s w}$, which is the enthalpy required to shock weaken the material. The constants in (12) are obtained from fitting the calculational results of Figure 8. The exponent is $-\mu / 2$, which is identical to the strength case.

The condition for the transition between the strength and the shock weakened regimes can be obtained by equating (11) and (12), which gives

$$
\frac{Y}{\rho H_{m}}=1.92
$$

A general relationship for depth that accounts for both regimes can be obtained by adding (11) and (12) which yields

$$
\frac{d_{p}}{a}=K_{s}^{d}\left(\frac{Y}{\rho U^{2}}\right)^{-0.28}+K_{s w}^{d}\left(\frac{H_{m}}{U^{2}}\right)^{-0.28}
$$




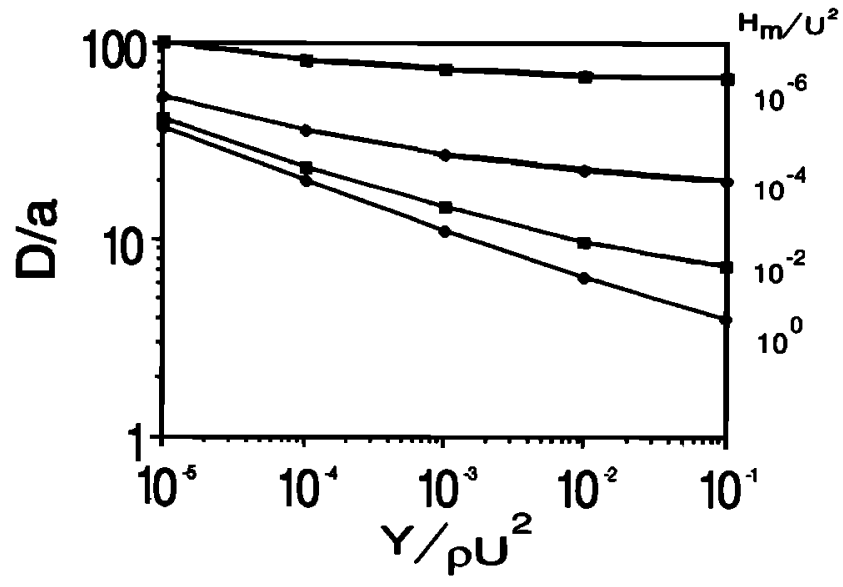

Fig. 9. Dimensionless crater diameter $(D / a)$ as a function of inverse Cauchy number $\left(Y / \rho U^{2}\right)$ in the strength dominated regime for various values of mell number.

where $K_{s}^{d}=0.84$ and $K_{s w}^{d}=0.7$. This relationship can be plotted over a range of values relevant to planetary surfaces and is shown in Figure 9. Equation (14) implies an effective strength which accounts for shock weakening which can be defined as

$$
Y_{e f f}=Y\left[1+\left(\frac{K_{s w}^{d}}{K_{s}^{d}}\right)\left(\frac{Y}{\rho H_{m}}\right)^{0.28}\right]^{-1 / 0.28}
$$

This result is plotted in Figure 10.

A general interpolation relationship of the form suggested by Holsapple and Schmidt [1987] can be used to span the gravity and strength regimes

$$
\begin{aligned}
& \frac{d_{p}}{a}=K_{s}^{d}\left(\frac{g a}{U^{2}}\right)^{-0.22} \\
& {\left[\left(\frac{K_{g}^{d}}{K_{s}^{d}}\right)^{-\frac{1}{0.22}}+\left(\frac{Y}{\rho U^{2}}\right)^{\frac{0.28}{0.22}}\left(\frac{g a}{U^{2}}\right)^{-1}\right]^{-0.22}}
\end{aligned}
$$

where $K_{g}^{d}=1.2$ (equation (9)).

Replacing the yield strength by the effective strength defined in (15) leads to a more general scaling relationship that includes gravity, strength and shock weakening effects

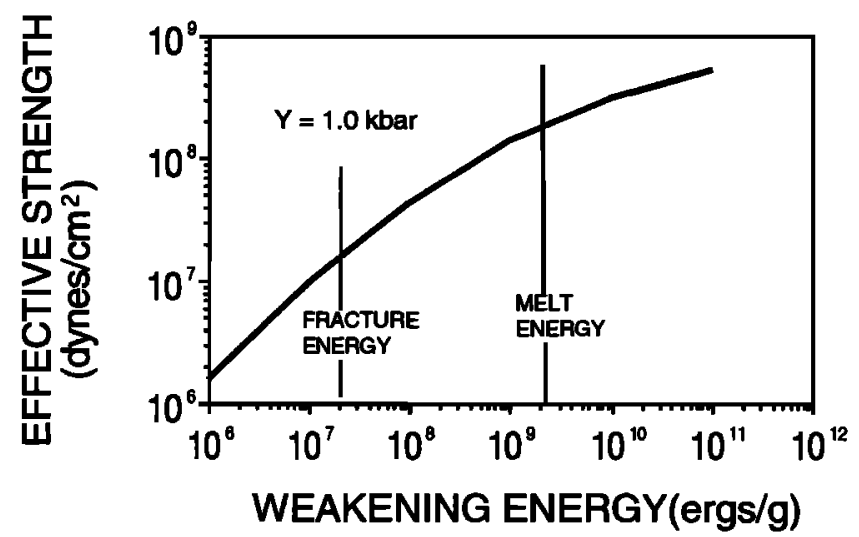

Fig. 10. Effective strength as a function of weakening energy.

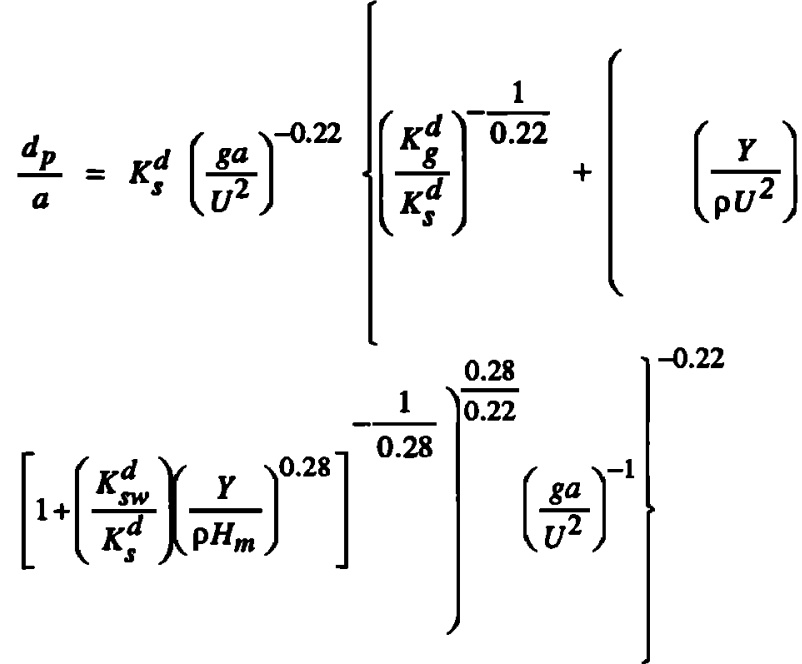

where the constants, $K$, were fit to code calculations in the different regimes.

The maximum depth of excavation of materials from giant impacts has been a topic of much debate in the literature [e.g., Grieve et al., 1981]. The tracer particles employed in the calculations help elucidate the motions of material in the cratering process. Referring to Figures 1, 2 , and 3 , we see that near the centerline of impact, planetary material is driven downward and is not ejected in the cratering process. As one moves from the centerline towards the crater lip, there is a region in which the planetary material is essentially stagnated against the outwardly moving cavity wall; this stagnated region, delineated by layers of tracer particles, shows a lower layer of particles being driven downward and an upper layer moving upward and being excavated (see Figures $1 c, 2 b$, $2 c$, and 3). The scaling laws for the maximum depth of excavation are given by

and

$$
\frac{d_{e x}}{a}=0.12\left(\frac{Y}{\rho U^{2}}\right)^{-0.28}
$$

$$
\frac{d_{e x}}{a} \cong 0.2\left(\frac{g a}{U^{2}}\right)^{-0.22}
$$

for the strength and gravity-dominated regimes. Here the exponents for strength and gravity scaling are given by (11) and (9). The proportionality constant is obtained from Figures 1 and 3.

\section{Diameter (D)}

The beginning of the terminal regime for the diameter occurs when the crater lip stops expanding laterally. In the case where the gravitational forces are dominant, this occurs when

$$
\frac{U t}{a}=1.8\left(\frac{g a}{U^{2}}\right)^{-0.61}
$$

where the magnitude of the exponent is given by $(1+\mu) /(2+\mu)$ and the proportionality factor in this equation and those of $(20)$ and (21), below, come from fitting the 
results of Figure 5. Note that this time is when the lip has stopped expanding laterally and just before it collapses into an outward propagating wave.

An example of the crater morphology near the time the crater cavity has reached its maximum diameter in the gravity dominated case is shown in Figures $2 c$ and $3 c$. This cavity maximum diameter is a function of the inverse Froúde number and is given by

$$
\frac{D_{m}}{a}=2.1\left(\frac{g a}{U^{2}}\right)^{-0.22}
$$

where the magnitude of the exponent is $\mu /(2+\mu)$. Note that the proportionality constant $(2.1)$ is intermediate in value between 2.0 for wet sand and 2.3 for water [Schmidt and Housen, 1987].

At the time the crater cavity has reached its maximum diameter, the crater lip has reached its maximum height (Figure 2c). This height scales as

$$
H_{\ell / a}=0.53\left(\frac{g a}{U^{2}}\right)^{-0.22}
$$

where the magnitude of the exponent is again $\mu /(2+\mu)$ and the proportionality factor was obtained from plots such as Figure $2 c$.

The crater lip will collapse and produce an outwardly propagating surface wave which rapidly increases the apparent diameter of the crater (Figures $2 c-2 d, 3 c, 3 d$ ). Simultaneously, the crater floor moves upward and an inner crater lip forms. The outer ring is defined as the arrested diameter of the enlarged crater which resulted from the collapse of the transient crater lip. Note that neither the outer ring nor the inner ring correspond to the maximum diameter of the transient crater. Figure 3 illustrates this point. The post-impact layering shows that the inner ring is much smaller than the transient crater diameter.

The diameter of the outer ring scales as

$$
\frac{D}{a}=4.28\left(\frac{g a}{U^{2}}\right)^{-0.22}
$$

where as before the magnitude of the exponent is given by $\mu /(2+\mu)$. The proportionality factor comes from Figure 5 . This final outer ring diameter is twice as large as the transient diameter. The transient diameter given by equation (20) is comparable with the transient diameters of water craters [Schmidt and Housen, 1987]. The final diameter is dependent upon the definition of which ring is assumed to be the crater diameter.

The observed crater morphologies in the gravity dominated regime are very rich in features which include single rings, multiple rings, central peaks, central pits, etc. The scaling of these features depends not only on gravity, but also on the mechanical and shock weakened properties of the planet. We have shown how a single outer ring is formed and how it scales with gravity, projectile size, and velocity. Other features of crater morphology are outside the scope of this paper will be reported upon elsewhere.
When strength dominates the cratering process, the crater growth ends in the inertial regime with only a small amount of elastic rebounding occurring followed by long term crater relaxation. The time of termination of crater formation is given by

$$
\frac{U t}{a}=0.33\left(\frac{Y}{\rho U^{2}}\right)^{-0.78}
$$

where the magnitude of an exponent is given by $(1+\mu) / 2$. Note that this time is identical to that for the depth. The parameters in (23) come from fitting the results of Figure 7.

The maximum or final crater diameter in the strength dominated regime is given by

$$
\frac{D}{a}=1.4 \quad\left(\frac{Y}{\rho U^{2}}\right)^{-0.28}
$$

where the exponent is $-\mu / 2$. Here we employed the results shown in Figure 8. In the shock weakened regime the final diameter is given by

$$
\frac{D}{a}=1.3\left(\frac{H_{m}}{U^{2}}\right)^{-0.28}
$$

where the exponent is $-\mu / 2$.

In a similar manner to the depth, a general interpolation relationship spanning the strength and shock weakened regimes is given by

$$
\frac{D}{a}=K_{s}^{D}\left(\frac{Y}{\rho U^{2}}\right)^{-0.28}+K_{s w}\left(\frac{H_{m}}{U^{2}}\right)^{-0.28},
$$

where for the diameter $K_{s}^{D}=1.4$ (equation (24)), and $K_{s w}$ $=1.3$ (equation (25)).

\section{Transition From Simple to Complex Craters}

The transition from simple to complex craters is the transition from strength-dominated to gravity-dominated craters. A general interpolation relationship for the crater diameter which spans both the gravity and strength regimes is given by

$$
\begin{aligned}
& \frac{D}{a}=K_{s}^{D}\left(\frac{g a}{U^{2}}\right)^{-0.22}\left\{\left(\frac{K_{g}^{D}}{K_{s}^{D}}\right)^{\frac{1}{0.22}}+()^{\frac{Y}{\rho U^{2}}}\right) \\
& \left.\left[1+\left(\frac{K_{s w}^{D}}{K_{s}^{D}}\right)\left(\frac{Y}{\rho H_{m}}\right)^{0.28}\right]^{\left.-\frac{1}{0.28}\right)^{\frac{0.28}{0.22}}\left(\frac{g a}{U^{2}}\right)^{-1}}\right\}^{-0.22}
\end{aligned}
$$




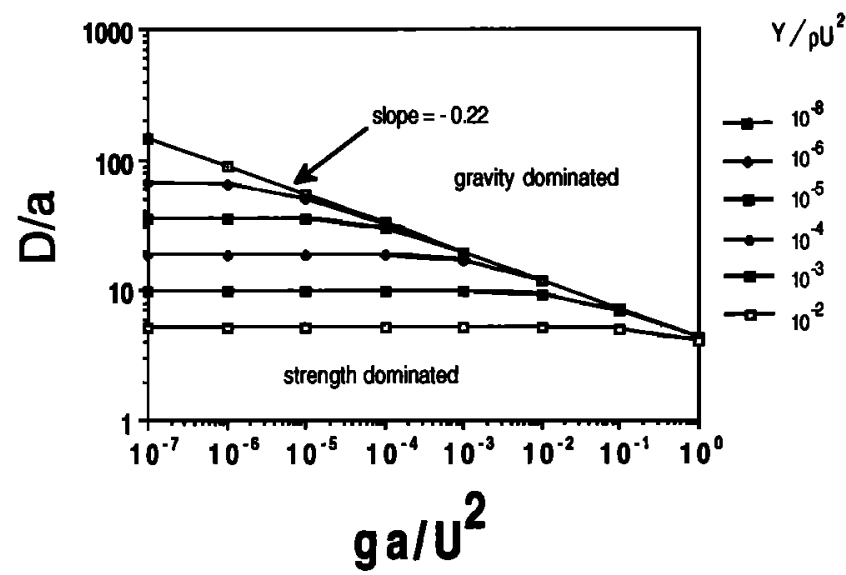

Fig. 11. Dimensionless crater diameter $(D / a)$ as a function of inverse Froude number $\left(g a / U^{2}\right)$ for various values of inverse Cauchy number $\left(Y / \rho U^{2}\right)$. Slope $(-0.22)$ corresponds to gravity scaling. Plot shows the transition from strength to gravity.

where for the diameter $K^{D}=1.4$ (equation (24)), and $K^{D}=1.3$ (equation (25)), and $K_{g}^{b}=4.28$ (equation (22)). This was obtained in the same manner as (17) for the depth of penetration. This result is plotted in Figure 11.

The transition diameter, or equivalently, the intersection between the strength and gravity dominated regimes can be readily derived. The transition diameter is obtained by equating $(20)$ for the gravity dominated transient diameter and strength dominated maximum diameter (24) and solving for the transition impactor radius. Substituting this radius into (24) yields the following simple relationship:

$$
D_{t}=K_{S}^{D}\left(\frac{K_{g}^{D}}{K_{S}^{D}}\right)^{\frac{2+\mu}{\mu}} \frac{Y}{\rho g}
$$

where $K_{g}^{D}$ is the gravity proportionality constant in equation (20) and $K_{S}^{D}$ is the strength proportionality constant in equation (24), and using those numerical values we obtain $K_{s}^{D}\left(K_{g}^{D} / K_{s}^{D}\right)^{(2+\mu) / \mu}=8.9$. Note that the transition diameter is directly proportional to the strength and inversely proportional to gravitational acceleration. This result is compared in Figure 12 to the transition diameters as a function of surface gravity for the terrestrial planets and various satellites [Chapman and McKinnon, 1986; Schenk, 1989].

The transition from simple to complex craters has been studied for some time using static models to predict the slumping of freshly formed craters [Melosh, 1977; Melosh and McKinnon, 1978]. Melosh [1977] found, using a perfectly plastic constitutive relation, that the criterion for stability of freshly formed bowl-shaped craters is given by

$$
\rho g d_{p} / Y=K_{m} \quad 5<K_{m}<10
$$

We will show that (29) can be cast in the form of (28) and leads to similar conclusions as those drawn by Melosh. Equation (29) can be solved for the maximum depth of penetration and that result is used in (11) to determine the transition impactor radius. Substituting this into (24) for the diameter yields

$$
D_{t}=K_{m}\left(\frac{K_{s}^{D}}{K_{s}^{d}}\right) \frac{Y}{\rho g}
$$

where $K_{s}^{d}$ is the strength proportionality constant in (11). Equating (28) and (30) gives the following expression for Melosh's proportionality constant:

$$
K_{m}=K_{s}^{d}\left(\frac{K_{g}^{D}}{K_{s}^{D}}\right)^{(2+\mu) / \mu}
$$

Note that equation (30) like equation (28) scales as $Y / P g$. For the numerical values found in our calculational results, we obtain a value of $K_{m}=5.3$, which is within Melosh's range of values $(5 \rightarrow 10)$.

\section{Shape, Observables, and Inferences}

In studying cratering on remote planetary surfaces, the observables are the surface morphology of the craters. In the following section we will relate some of the transient nonobservable aspects of the cratering process (e.g., depth of penetration, excavation, lip height) to the usual observable quantity, crater diameter.

The maximum transient cavity diameter relative to the final crater diameter in gravity dominated craters is given by dividing $(20)$ by $(22)$, which yields

$$
\frac{D_{m}}{D}=0.5
$$

and for strength dominated craters, the maximum cavity diameter is equal to the final diameter, except for minor crater rebounding. Notably, the maximum transient diameter is the maximum diameter in this case,

$$
\frac{D_{m}}{D}=1
$$

The maximum crater lip height relative to the crater diameter in gravity dominated craters can be obtained by dividing (21) by (22) to get

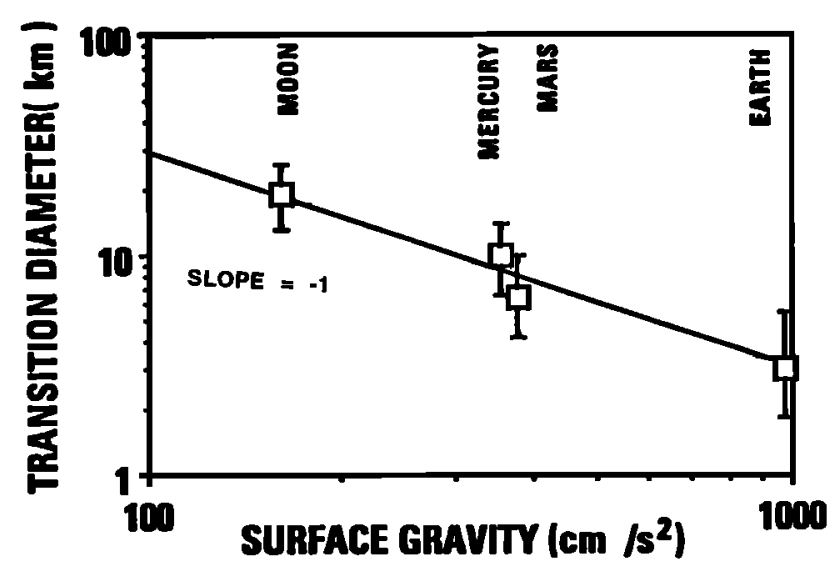

Fig. 12. Crater transition diameter $\left(D_{t}\right)$ from simple to complex craters as a function of planetary surface gravity. Data are from Schenk [1989]. Slope corresponds to scaling law of (28). 


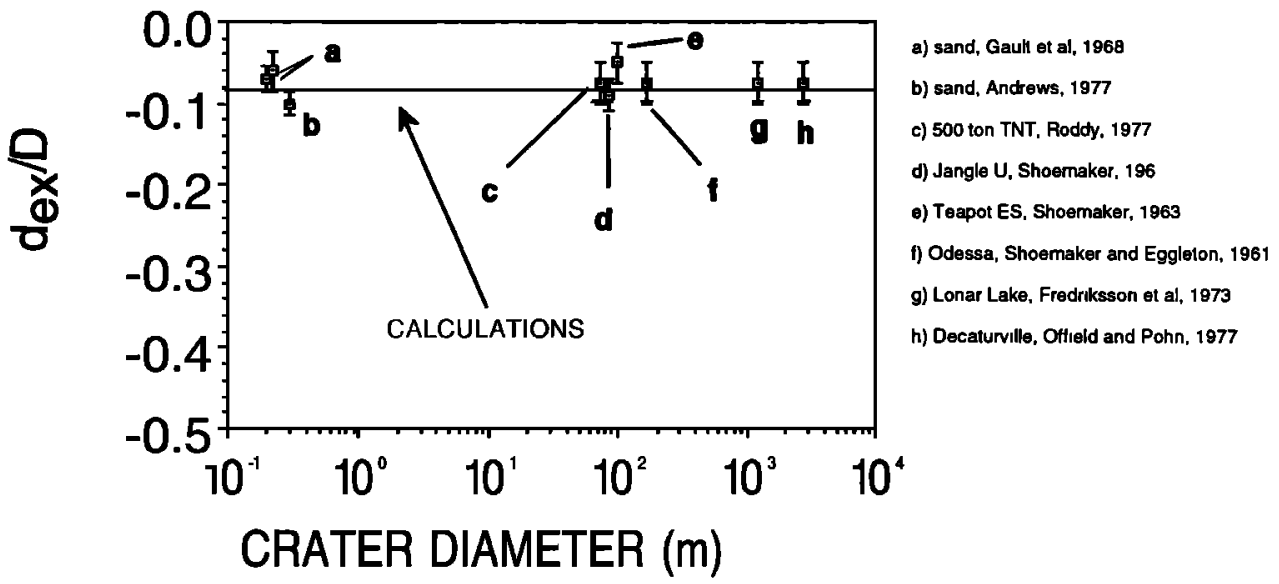

Figure 13. Depth of excavation of planetary material $\left(d_{e x}\right)$ divided by the crater diameter $(D)$ as a function of crater diameter for terrestrial craters. Calculation results indicated by solid line. (Point a) Sand [Gault et al., 1968], (point b) sand [Andrews, 1977], (point c) 500 ton TNT [Roddy, 1977], (point $d$ ) Jangle U [Shoemaker, 1963], (point $e$ ) Teapot Ess [Shoemaker, 1963], (point $f$ Odessa [Shoemaker and Eggleton, 1961], (point \&) Lonar Lake [Fredriksson et al., 1973], and (point $h$ ) Decaturville [Offield and Pohn, 1976].

$$
\frac{H_{l}}{D}=0.12
$$

The ratio of the time for the crater lip to collapse $\left(t_{l p}\right)$ to the time for the crater floor to rebound $\left(t_{r}\right)$ in the gravity dominated regime can be obtained from Figure 5 and is given by

$$
\frac{t_{l p}}{t_{r}} \cong 1.8
$$

The depth of penetration relative to the crater diameter for gravity-dominated craters can be obtained by dividing (9) by (22), which yields

$$
\frac{d_{p}}{D}=0.28
$$

and for strength dominated craters by dividing (11) by (24), which yields

$$
\frac{d_{p}}{D}=0.6
$$

In a similar manner, the depth of excavation relative to the crater diameter for gravity dominated craters can be obtained by dividing ( $18 b$ ) by (22), which yields

$$
\frac{d_{e x}}{D}=0.05
$$

and for strength dominated craters by dividing (18a) by (24):

$$
\frac{d_{e x}}{D}=0.085
$$

Note that the depth of penetration and excavation relative to the crater diameter are a factor of two less in the gravity dominated cases because the crater diameter is larger as a result of the lip collapse and lip wave propagation. The above result for the depth of excavation is compared to field data from terrestrial craters in Figure 13. The maximum depth of penetration and depth of excavation as a function of crater diameter are plotted for the various planets in Figure 14. As an example, for craters in the range of $150 \mathrm{~km}$ in diameter, which is in the estimated size for the $K-T$ event [Alvarez et al., 1980], the maximum depth of penetration is $-42 \mathrm{~km}$ while the maximum depth of excavation is $-13 \mathrm{~km}$. Impacts of this magnitude appear to open the possibility of some impactinduced volcanism from the Earth's mantle.

\section{CONCLUSIONS}

The objective of this study was to obtain a quantitative understanding of the cratering process over a broad range of conditions. In accomplishing this objective we numerically computed the evolution of impact induced flow fields and calculated the time histories of the major measures of crater geometry (e.g. depth and diameter) for variations in planetary gravity, material strength, and impactor size. The results of these calculations are summarized in Table 2 . These results were fit with the scaling laws of Holsapple and Schmidt [1987] and compared to experimental results of Schmidt and Housen [1987]. In all of the measures we examined, these scaling laws were found to be in good agreement with the numerical results. From this experience, we expect this agreement to hold for other crater descriptions. The exceptions are the amount of melting and vaporization, which are dominated by shock heating during the penetration regime.

We have described the impact process in terms of four regimes: (1) penetration, (2) inertial, (3) terminal , and (4)

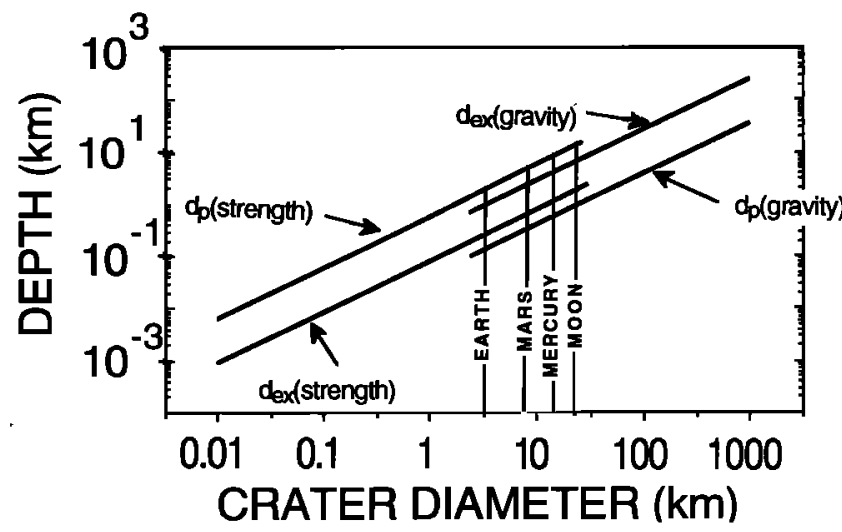

Fig. 14. Maximum depth of penetration $\left(d_{p}\right)$ and excavation $\left(d_{e x}\right)$ as a function of crater diameter $(D)$ for various planets and satellites. Vertical lines delineate the transition from strength to gravity scaling. For gravitydominated craters, the diameter is defined as the outer ring. 

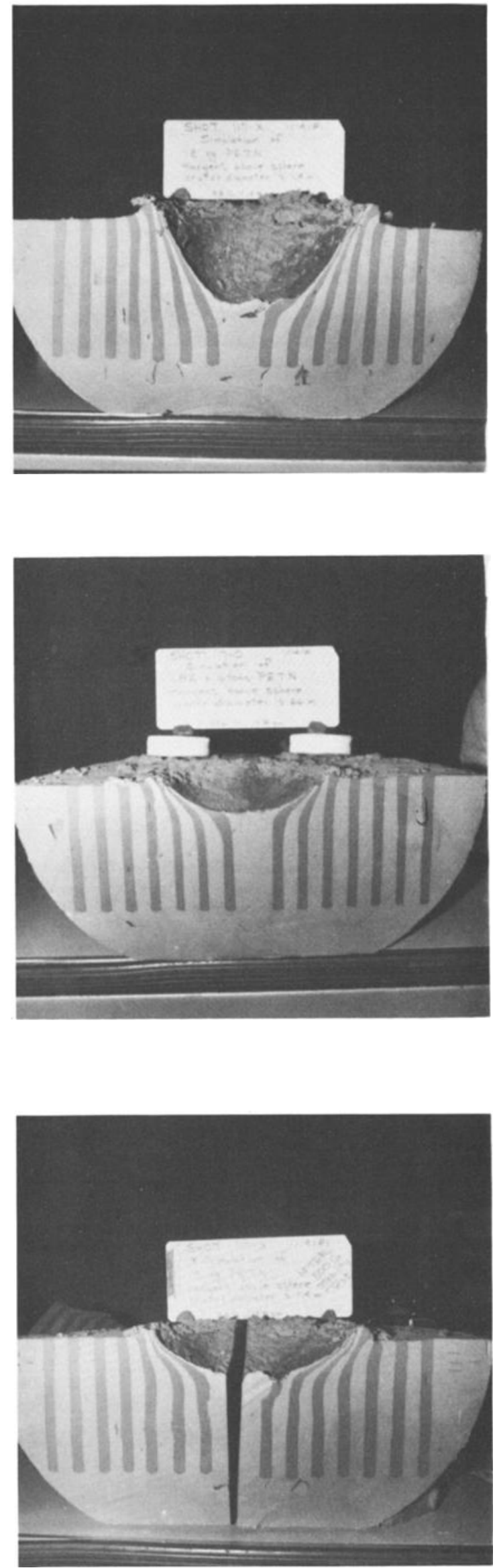

relaxation. The scaling laws for each of the first three regimes are summarized in Table 3 . In the penetration regime the depth evolves linearly with time. In the inertial regime, the scaling of the crater geometry is a function of the coupling exponent $(\mu)$ as defined by Holsapple and Schmidt [1987] and was shown by them to be related to the slope of the growth of the depth and diameter in that regime. The results of this paper confirm that relationship.

The value found for the coupling exponent, $\mu$, was 0.56 which is consistent with range of values found by Holsapple and Schmidt [1987]. This value implies that the volume of the crater scales in between energy and momentum scaling (e.g. $\mu=2 / 3$ for energy scaling, and $\mu$ $=1 / 3$ for momentum scaling). During the penetration regime ( $\tau \leq 5.1$, the phenomena associated with the crater evolution such as the volume of rock melted and vaporized scale as the energy of impact. However for $\tau>5$, some of the shocked material is ejected from the growing transient cavity. This reduces the energy in the cavity and causes the shock wave to decay more rapidly. This loss of energy from the cavity results in the scaling of descriptors of the flow, such as particle velocity, in the inertial regime to the lower than predicted from energy scaling but somewhat greater than predicted from momentum scaling.

In studying cratering remotely on planetary surfaces, the primary observables are the crater diameter including the diameters of the multiple rings, the presence of central peaks and pits and the relaxed crater depth. We have related several key transient nonobservable aspects of the cratering process (e.g. maximum depth of penetration, depth of excavation, and maximum crater lip height) to the crater diameter. For example, the maximum depth of penetration relative to the crater diameter is -0.6 for strength dominated craters and $\mathbf{- 0 . 3}$ for gravity dominated craters. This implies that impactors associated with the large basin impacts penetrated to great depths on the planets. However, detailed observations of terrestrial impact craters demonstrate that the stratigraphy, both below and surrounding the craters, appear to be undisturbed. This has been erroneously used to imply that the impactor did not penetrate deeply. However, laboratory experiments under high accelerations [e.g. Schmidt and Housen, 1987, Figure 10] simulating large scale impacts also exhibit intact layering sequences at relatively shallow depth (Figure 15). In examining the details of the rebounding of the depth and the collapse and propagation of the crater lip in the gravity dominated regime, we observe that the initial target stratigraphy is reconstructed in the rebound process. In Figure 3, we have connected these particles to clearly illustrate this effect. Moreover, in the laboratory tests of Schmidt and Holsapple [1987], they produced a crater at low acceleration (simple bowl-shaped crater) and subjected it to high acceleration (522 $\mathrm{ge}^{\mathrm{s}} \mathrm{s}$ ) and found that it rebounded and nearly perfectly reconstructed the initial

Fig. 15. Laboratory scale craters at low and high acceleration levels that illustrate the reconstruction of the target stratigraphy [after Schmidt and Housen, 1987]. (a) Crater produced in clay with 12.8 \& PEIN explosive (tangent above) under $10 \mathrm{~g}$ conditions and is strength dominated. (b) Gravity-dominated crater formed at $526 \&$ (corresponding to 1.86 ktons of PETN at 18 ). The depth is shallow and the layering sequence is undeformed at relatively shallow depths. (c) The crater formed at $10 \mathrm{~g}$ (a) that was subjected to $522 \mathrm{~g}$ and illustrates the collapse and reconstruction of the layering sequence. The dark vertical bisecting zone is an artifact of dissection. 
stratigraphy (Figure 15). In examining the details of the rebounding of the crater in the gravity dominated regime, we found that the initial planetary material layering sequence is reconstructed in the rebound process (see Figure 3).

In a similar manner to the penetration, the maximum deep of excavation relative to the crater diameter was examined and found to be $\sim 0.05$ for gravity dominated craters and $\mathbf{~} 0.09$ for strength dominated; this implies that for impact velocities less than the threshold for significant vaporization, the excavated material comes from relatively shallow depths. However, for very large craters the absolute depth of excavation could be significant, and could give rise to impact induced volcanism.

The transition from simple bowl shaped craters to complex craters was analyzed. This occurs when gravity starts to dominate over strength in the terminal phase. The transition diameter is proportional to $\mathrm{Y} / \mathrm{\rho g}$ where the proportionality constant is -9.0 .

Acknowledgments. Research supported under NASA grant NAGW 1953. We appreciate the computational assistance of $M$. Lainhart and many helpful discussions with $K$. Holsapple and $R$. Schmidt. We very much appreciate the thoughtful and detailed comments made by the reviewers and especially the detailed comments and suggestions of $\mathbf{R}$. Schmidt. In addition, we would like to thank $R$. Schmidt for photocopies of the figure from his paper. Division of Geological and Planetary Sciences, California Institute of Technology, Pasadena, contribution 4859.

\section{REFERENCES}

Ahrens, T. J., and A. Rubin, Impact-induced tensional failure in rock, J. Geophys. Res., 98, 1185-1203, 1993.

Alvarez, L. E., W. Alvarez, F. Asaro, and H. V. Michel, Extraterrestrial cause for the Cretaceous-Tertiary extinction, Science, 208, 1095-1108, 1980.

Andrews, R. J., Characteristics of debris from small-scale cratering experiments, in Impact and Explosion Cratering, edited by D. J. Roddy, R. O. Pepin and R. B. Merrill, pp. 1089-1100, Pergamon, New York, 1977.

Asphaug, E., H. J. Melosh, and E. Ryan, A numerical laboratory for fragmentation studies: Some insights into collisional processes and outcomes (abstract), Lunar Planet. Sci., XXII, 37-38, 1991.

Bjork, R. L., Analysis of the formation of Meteor Crater, Arizona. A preliminary report, J. Geophys. Res., 66, 3379-3387, 1961.

Bjorkman, M. D., and K. A. Holsapple, Velocity scaling of impact melt, Int. J. Impact Eng., 5, 155-163, 1987.

Chapman, C. R., and W. B. McKinnon, Cratering of planetary satellites, in Satellites, edited by J. A. Burns and M. S. Mathews, Pp. 492-580, Univ. Arizona Press, Tucson, AZ, 1986.

Frederiksson, K., A. Duba, D. J. Millon, and M. S. Balasundaram, Lonar Lake, India: An impact crater in basalt, Science, 180, 862 864, 1973.

Gault, D. E., and C. P. Sonnett, Laboratory simulation of pelagic asteroidal impact: Atmospheric injection, benthic topography, and surface wave radiation, Geol. Soc. Am. Spec. Pap. 190, 64-91, 1982.

Gault, D. E., W. L. Quaide, and V. R. Oberbeck, Impact cratering mechanics and structures, in Shock Metamorphism of Natural Materials, edited by B. M. French and N. M. Short, pp. 87-100, Mono Press, Baltimore, MD, 1968.

Grieve, R. A. F., P. B. Robertson, and M. R. Dence, Constraints on the formation of ring impact structures, based on terrestrial data, in Multi-Ring Basins, edited by P. H. Schultz and R. B. Merrill, pp. 3757. Pergamon, New York, 1981.

Holsapple, K. A., and R. M. Schmidt, Point-source solutions and coupling parameters in cratering mechanics, J. Geophys. Res., 92, 6350-6376, 1987.

Housen, K. R., and K. A. Holsapple, On the fragmentation of asteroids and planetary satellites, Icarus, 84, 220-253, 1990.

Housen, K. R., R. M. Schmidt, and X. A. Holsapple, Crater ejecta scaling laws: Fundamental forms based on dimensional analysis, $J$. Geophys. Res., 88, 2485-2499, 1983.
Kieffer, S. W., Impact conditions Required for melt by jetting in silicates. in Impact and Explosion Cratering, edited by D. J. Roddy, R. O. Pepin, and R. B. Merrilll, pp. 751-770, Pergamon, New York, 1976.

Melosh, H. J., Crater modification by gravity: A mechanical analysis of slumping, in Impact and Explosion Cratering, edited by D. J. Roddy, R. O. Pepin, and R. B. Merrill, pp. 1245-1260, Pergamon, New York, 1977.

Melosh, H. J., Impact Cratering, A Geologic Process, pp. 1-245, Oxford University Press, New York, 1989.

Melosh, H. J., and W. B. McKinnon, The mechanics of ringed basin formation, Geophys. Res. Lett., 5, 985-988, 1978.

Offield, T. W., and H. A. Pohn, Deformation at the Decaturville impact structure, Missouri, in Impact and Explosion Cratering, edited by D. J. Roddy, R. O. Pepin and R. B. Merrill, pp. 321-341, Pergamon, New York, 1977.

O'Keefe, J. D., and T. J. Ahrens, Impact induced energy partitioning, melting, and vaporization on terrestrial planets, Proc. Lunar Sci. Conf., 8th, 3357-3374, 1977.

O'Keefe, J. D., and T. J. Ahrens, Large scale impact on the earth with an atmosphere (abstract), Lunar Planet. Sci., XIX, 887-888, 1988.

O'Keefe, J. D., and T. J. Ahrens, The effects of gravity, size, and velocity on the scaling of crater geometry and the transition from simple to complex craters (abstract), Lunar Planet. Sci., XX, 810811, 1989.

O'Keefe, J. D., and T. J. Ahrens, Impact melting on planetary surfaces, in Proceedings of 1992 Sudbury Conference on Large Body Impacts on Planetary Evolution, edited by B. Dressler and R. V. Sharpton, Geol. Soc. Am. Spec. Pap., Boulder, CO, in press, 1993.

Roddy, D. J., Large-scale impact and explosion craters: Comparisons of morphological and structural analogs, in Impact and Explosion Cratering, edited by D. J. Roddy, R. O. Pepin and R. B. Merrill, pp. 185-246, Pergamon, New York, 1977.

Roddy, D. J., S. H. Schuster, M. Rosenblatt, L. B. Grant, P. J. Hassig, and $K$. N. Kreyenhagen, Computer simulations of large asteroid impacts into oceanic and continental sites---Preliminary results on atmospheric, cratering and ejecta dynamics, Int. J. Impact Eng., 5, 525-541, 1987.

Schenk, P. M., Crater formation and modification on the icy satellites of Uranus and Saturn: Depth/diameter and central peak occurrence, J. Geophys. Res., 94, 3813-3832, 1989.

Schmidt, R. M., A centrifuge cratering experiment: Development of a gravity-scaled yield parameter, in Impact and Explosion Cratering. edited by D. J. Roddy, R O. Pepin, and R. B. Merrill, pp. 12611278, Pergamon, New York, 1977.

Schmidt, R. M., Meteor crater: Energy at formation - Implication of centrifuge scaling, Proc. Lunar Planet. Sci. Conf., 11th, 2099-2128, 1980.

Schmid, R. M., and K. R. Housen, Some recent advances in the scaling of impact and explosion cratering, Int. J. Eng. 5, 543-560, 1987.

Schultz, P. H., and D. E. Gault, Impact ejecta dynamics in an atmosphere: Experimental results and extrapolations, Geol. Soc. Am. Spec. Pap. 190, 152-174, 1981.

Shoemaker, E. M., Impact mechanics at Meteor Crater, Arizona, in Moon, Meteorites, and Comets, edited by B. M. Middlehurst and G. P. Kuiper, pp. 301-336, University of Chicago Press, Chicago, Ill., 1963.

Shoemaker, E. M., and R. E. Eggleton, Terrestrial features of impact origin, Proceedings, Geophysical Lab, Lawrence Radiation Laboratory Cratering Symposium, Rep. UCRL-6438, Lawrence Radiation Lab., Livermore, CA, 1961.

Simmons, G., T. Todd, and H. Wang, The $25-\mathrm{km}$ discontinuity: Implications for lunar history, Science, 182, 158-161, 1973.

Thompson, S. L., CSQII - An Eulerian finite-difference program for two-dimensional material response - Part 2. Energy flow sections, Sandia National Labs, Albuquerque, N.M., Rep. SAND 77-1340, Sandia Natl. Labs, Albuquerque, N.M., 1979.

T. J. Ahrens and J. D. O'Keefe, Lindhurst Laboratory of Experimental Geophysics, Seismological Laboratory, California Institute of Technology, Pasadena, CA 91125.

(Received February 19, 1992; revised May 19, 1993; accepted May 20, 1993.) 